\title{
MATHEMATICAL AND NUMERICAL MODELING OF SOLUTE DYNAMICS IN BLOOD FLOW AND ARTERIAL WALLS*
}

\author{
ALFIO QUARTERONI ${ }^{\dagger \ddagger}$, ALESSANDRO VENEZIANI ${ }^{\ddagger}$, AND PAOLO ZUNINO $^{\dagger}$
}

\begin{abstract}
The numerical modeling of solutes absorption processes by the arterial wall is of paramount interest for the understanding of the relationships between the local features of blood flow, the nourishing of the inner arterial wall by the blood solutes, and the pathologies that can appear when this process is for some reason perturbed. In the present work, two models for the solutes dynamics are investigated. In the first model, which is essentially based on the one introduced by Rappitsch and Perktold [J. Biomech. Engrg., 118 (1996), pp. 511-519] and Rappitsch, Perktold, and Pernkopf [Internat. J. Numer. Methods Fluids, 25 (1997), pp. 847-857], the Navier-Stokes equations for an incompressible fluid, describing the blood velocity and pressure fields, are coupled with an advection-diffusion equation for the solute concentration. The wellposedness of this model is discussed. The second model considers also the solutes dynamics "inside" the arterial wall, described by a pure diffusion equation. Actually, this is a heterogeneous model, coupling different equations in different parts of the domain at hand. Its wellposedness is proven. Moreover, in view of the numerical study, an iterative finite element method by subdomains is proposed and its convergence properties are analyzed. Finally, several numerical results comparing the different models in situations of physiologic interest are illustrated.
\end{abstract}

Key words. computational hemodynamics, mass transfer processes, coupling of heterogeneous models, finite element methods

AMS subject classifications. 76D05, 76Z05, 76R50, 76M10, 65M60, 65Y99

PII. S0036142900369714

\section{Introduction.}

Motivations. The major task of blood is to provide oxygen and nourishment to the tissues and the organs and to collect waste substances, such as carbon dioxide. Different substances are therefore dissolved in blood and exchanged in different districts. In particular, the inner part of the arterial wall (the so-called "intima") is involved by transport phenomena of blood solutes. These phenomena are essential for the correct nourishment of these tissues and are possibly related to the development of some diseases such as atherosclerosis (see, e.g., $[2,4,26,27]$ ). The dynamics of blood solutes are strongly related to the dynamics of blood. In fact, the solutes are essentially convected by the blood along the vessels. The absorption processes through the arterial wall are related to the stress induced by the blood on the vascular tissue (see, e.g., $[2,9,26,27]$ ). Finally, the particular nature of the blood, which is a suspension of many particles, is at the origin of a diffusivity enhancement of some kind of gaseous solutes, such as oxygen. More precisely, a localized stirring caused by the rotation of red blood cells produces a solute diffusivity augmentation, which is definitely a function of the shear rate of blood (see [12, 30, 31, 3]). The numerical simulation of solutes dynamics inside the vessels as well as into the vascular walls

*Received by the editors March 7, 2000; accepted for publication (in revised form) May 17, 2001; published electronically December 26, 2001. This work was supported by the FNRS Swiss grant 21- 59230.99 and by Gruppo Nazionale di Informatica Matematica-GNIM Project "Modelling and Simulation of Fluid Equations and Mass Transport Processes in Heterogeneous Media."

http://www.siam.org/journals/sinum/39-5/36971.html

†Départément de Mathématiques, École Polytechnique Fédérale de Lausanne, CH-1015, Lausanne, Switzerland (alfio.quarteroni@epfl.ch, paolo.zunino@epfl.ch).

‡Dipartimento di Matematica "F. Brioschi," Politecnico di Milano, Piazza Leonardo da Vinci 32, I-20133 Milano, Italy (alfqua@mate.polimi.it, ales@mate.polimi.it). 
could therefore be useful for revealing the relationships between local flow patterns in a district, absorption, and possible pathogenesis (see, e.g., [26]).

Problem formulation and analysis. In this paper we consider two different models for the dynamics of solutes in the arteries. At first, we consider the model discussed in [26] and [27] for the oxygen and lipoprotein absorption processes. In this model, the Navier-Stokes equations for an incompressible fluid are adopted to describe the blood motion and are coupled with an advection-diffusion equation, modeling the solute dynamics. The velocity of blood provides the convective field of the solute equation. Moreover, it is supposed that the permeability governing the absorption process of the solute through the wall is a suitable function of the shear stress exerted by the blood on the arterial wall; in mathematical terms, this means that the boundary conditions for the solute equation on the arterial wall are again related to the blood velocity field. Finally we assume that the diffusion of the solute is represented by a diffusivity tensor which is a function of the shear rate. In this model we neglect the solute dynamics "inside" the arterial wall, and therefore, for the sake of convenience, we refer to it as the wall-free model. The wellposedness of this model is investigated.

Next, we consider an extension of this model, also taking into account the dynamics of solutes inside the vascular tissue (see $[10,11]$ ). The convective field inside the wall is small and can be neglected; therefore the dynamics of solutes are described by a pure diffusion equation. This equation is coupled to the advection-diffusion model of the "fluid-side" by means of suitable boundary conditions, prescribing the continuity of the normal solute fluxes. Since the arterial wall plays the role of a selective permeable membrane, the continuity of the solute concentration across the membrane is not prescribed at all, but a kind of "constitutive law" for the membrane behavior is considered. The wellposedness analysis of this second model, given by the coupling of Navier-Stokes equations, an advection-diffusion equation in the fluid or lumen side, and a diffusion equation in the wall side, is also carried out. We will refer to this model as the fluid-wall one.

Numerical validation and assessment. The numerical treatment of the problems associated with both the considered models is carefully discussed. For all the involved equations, in the lumen as well as in the wall domain, the space discretization is based on the finite element method, while the time discretization is carried out by means of classical finite difference schemes. The time discretization of the Navier-Stokes equations, in particular, is carried out by the Yosida method that allows a suitable splitting of the velocity and pressure computation (see [20,21]). Moreover, since the fluid-wall model is actually a heterogeneous model, coupling different problems in different parts of the computational domain at hand, it is worthwhile to introduce an iterative procedure for solving alternatively two problems in the two subdomains. (This provides a special instance of iterative substructuring approach; see, e.g., [25].) Different iteration strategies can be carried out; although they all provide the same limit solution, their numerical efficiency (i.e., convergence rate) can be quite different. Here, we consider in particular a strategy based on Robin-like interface conditions both for the fluid and the wall sides of the domain. This choice is mathematically justified and extremely effective. In particular, we prove the convergence of the solution computed by the adopted subdomains method to the solution of the unsplit problem. The rate of convergence will be proven to be independent of the space discretization size. Finally, several numerical results concerning the wall-free as well as the fluid-wall models are shown in physiologically relevant situations.

The paper is organized as follows. In section 2 we introduce the mathematical 
formulation of wall-free and fluid-wall models, discussing their relevant features. In section 3 we prove the wellposedness of the associated weak problems. In section 4 we describe the numerical solution techniques for the two models: in particular, in section 4.3, the convergence analysis of the iterative substructuring method adopted for the fluid-wall model is carried out. Finally, numerical results on data of clinical interest are addressed in section 5 .

\section{Description of the models.}

2.1. The wall-free model. Let us denote by $\Omega_{f} \subset \mathbb{R}^{d}(d=2,3)$ the lumen of a given vascular district. The boundary of $\Omega_{f}$ is composed by different parts, namely the proximal sections $\Gamma_{u p}$, that is, the upstream part (with respect to the heart and the blood flow) of the vascular district; the distal sections $\Gamma_{d w}$, which delimit the district downstream; the part of $\partial \Omega_{f}$ corresponding to the arterial wall is denoted by $\Gamma_{w}$, so that $\partial \Omega_{f}=\Gamma_{w} \cup \Gamma_{u p} \cup \Gamma_{d w}$ (see Figure 2.1). Different boundary conditions can be chosen for the fluid and the solute dynamics on $\Gamma_{u p}$ and $\Gamma_{d w}$; for the mathematical analysis, without losing generality, we consider (homogeneous) Dirichlet boundary conditions. Concerning the blood motion, we will introduce the following hypotheses: (a) the blood is a Newtonian fluid, i.e., we assume that blood is a fluid with a constant (kinematic) viscosity denoted by $\nu$; (b) the arterial wall is rigid. The first hypothesis is actually reasonable in large and medium size arteries (see, e.g., [22]). The second hypothesis relies on the experimental observation of small displacements for the vascular walls "in vivo." The extension of the results of the present work to the case of compliant walls will be the subject of a forthcoming paper.
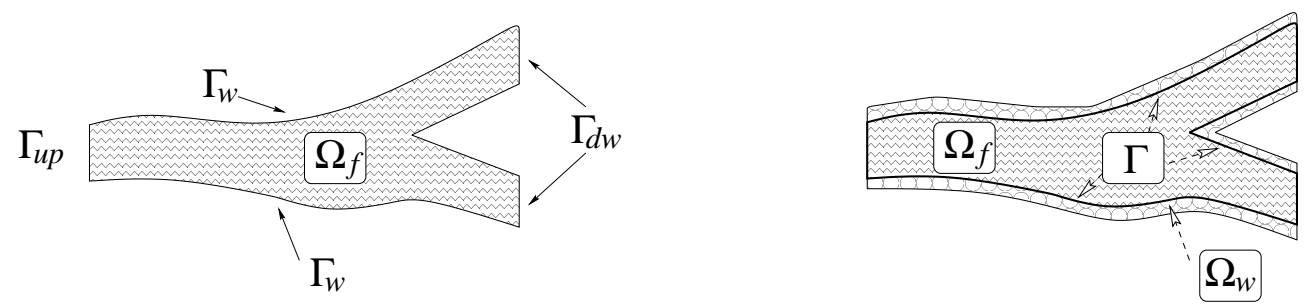

FIG. 2.1. Scheme of the wall-free (left) and the fluid-wall (right) models.

In what follows, for $\mathbf{x} \in \Omega_{f}$ and $t>0, \mathbf{u}(t, \mathbf{x}) \in \mathbb{R}^{d}$ will denote the velocity field of the blood, and $P(t, \mathbf{x})$ the kinematic pressure, i.e., the ratio between the pressure and the blood density. Moreover, $C_{f}(t, \mathbf{x})$ will denote the concentration of the considered solute (e.g., $\mathrm{O}_{2}$ or lipoproteins) in the blood. Finally, $\kappa_{w}(t, \mathbf{x})$, for $\mathbf{x} \in \Gamma_{w}$, denotes the solute concentration on the arterial wall $\Gamma_{w}$. In this model, $\kappa_{w}$ is a given quantity.

Remark 2.1. The choice of the concentration as the unknown of the advectiondiffusion problem is questionable. Actually, when gaseous solutes are involved, one could also write the equations in terms of the (partial) pressure of the solute, since in this way it is more immediate to consider different forms of the same solute (such as oxygen, which is present both as free solute in plasma and as a form linked to hemoglobin). By means of approximated empirical laws, the relationship between partial pressure and concentration is assumed to be linear (see, e.g., [18]). Hence, from the mathematical viewpoint, the associated models are very similar, and we will refer to the solute unknown simply as "concentration." For the same reason, in our analysis we limit ourselves to consider the presence of just one solute. In blood 
there are indeed many solutes; however, the mathematical analysis of the multisolute situations is not significantly more involved than in the case with one solute.

The wall-free model is defined through the two following systems.

Problem 2.1.

$$
\left\{\begin{array}{l}
\frac{\partial \mathbf{u}}{\partial t}+(\mathbf{u} \cdot \nabla) \mathbf{u}-\nu \Delta \mathbf{u}+\nabla P=\mathbf{f}, \quad \mathbf{x} \in \Omega_{f}, \quad t>0, \\
\nabla \cdot \mathbf{u}=0, \quad \mathbf{x} \in \Omega_{f}, \quad t>0, \\
\mathbf{u}=\mathbf{b} \quad \text { on } \quad \partial \Omega_{f} \backslash \Gamma_{w}, \quad \mathbf{u}=\mathbf{0} \quad \text { on } \quad \Gamma_{w}, \quad t>0, \\
\mathbf{u}=\mathbf{u}_{0} \quad \text { with } \quad \nabla \cdot \mathbf{u}_{0}=0, \quad \mathbf{x} \in \Omega_{f}, \quad t=0 .
\end{array}\right.
$$

Problem 2.2.

$$
\left\{\begin{array}{l}
\frac{\partial C_{f}}{\partial t}-\nabla \cdot\left(\boldsymbol{\mu}_{f} \nabla C_{f}\right)+\mathbf{u} \cdot \nabla C_{f}=f_{f}, \quad \mathbf{x} \in \Omega_{f}, \quad t>0 \\
\text { (a) } \mathbf{n} \cdot\left(\boldsymbol{\mu}_{f} \nabla C_{f}\right)+\zeta C_{f}=\zeta \kappa_{w} \quad \text { on } \quad \Gamma_{w}, \quad t>0, \\
\text { (b) } \quad C_{f}=0 \quad \text { on } \quad \partial \Omega_{f} \backslash \Gamma_{w}, \quad t>0, \\
C_{f}=C_{f, 0}, \quad \mathbf{x} \in \Omega_{f}, \quad t=0 .
\end{array}\right.
$$

Recall that in this model the vessel wall is assumed to be rigid. The boundary condition (2.2a) states that the flux of solute entering or leaving the blood domain through $\Gamma_{w}$ is related to the difference of concentration across the boundary by the arterial wall permeability $\zeta$. The diffusivity tensor $\boldsymbol{\mu}_{f}$ is a function of the rate of deformation tensor $\mathbf{d}$, whose components are

$$
d_{i j}:=\frac{1}{2}\left(\frac{\partial u_{i}}{\partial x_{j}}+\frac{\partial u_{j}}{\partial x_{i}}\right), \quad i, j=1,2, \ldots, d .
$$

Then, by experimental observations (see $[30,31,1]$ ), it has been proposed that

$$
\left(\mu_{f}\right)_{i j}:=\mu_{f 0}\left(\delta_{i j}+\mathcal{D}\left|d_{i j}\right|^{\epsilon}\right), \quad i, j=1,2, \ldots, d,
$$

where the positive coefficients $\mu_{f 0}, \mathcal{D}$, and $\epsilon$ depend on the specific solute and on the concentration of red blood cells, which is assumed to be constant; $\delta_{i j}$ denotes the Kronecker identity tensor.

Similarly, the wall permeability $\zeta$ is modeled as a function of the shear stress $\boldsymbol{\sigma}(\mathbf{u})$ induced by the blood flow on the arterial wall (see [26, 27]). We recall that $\boldsymbol{\sigma}(\mathbf{u})=\boldsymbol{\tau} \cdot \mathbf{T}(\mathbf{u}) \cdot \mathbf{n}$, where $\mathbf{T}(\mathbf{u})$ is the local stress tensor defined as $\mathbf{T}(\mathbf{u})=2 \nu \mathbf{d}$ and $\mathbf{n}, \boldsymbol{\tau}$ are the normal and the tangential unit vectors on $\Gamma_{w}$, respectively. More precisely it is assumed that $\zeta=\zeta(|\boldsymbol{\sigma}(\mathbf{u})|)$, where $\zeta$ is a positive Lipschitz continuous function; i.e., there exists a constant $L$ such that $0 \leq \zeta(x) \leq L|x|$ for each value of the argument $x$. Here, we will assume that $\zeta>0$ on a subset of $\Gamma_{w}$ with nonzero measure. This hypothesis is obviously necessary in order to have a nonnull outgoing solute flux through the arterial wall, which is exactly the process we wish to investigate. In [26] and [27] some models for the dependence of $\zeta$ on $\boldsymbol{\sigma}(\mathbf{u})$ are proposed for various types of solutes. We will provide an example in section 5 . 
Finally, $\mathbf{f}$ and $f_{f}$ represent possible forcing terms for the blood velocity and the solute concentration, respectively. More precisely, f could be the gravity force, while $f_{f}$ could represent the effect of chemical reactions of the solute with other substances dissolved in blood.

Referring to condition (2.2b), we point out that it is not restrictive to consider homogeneous Dirichlet boundary conditions because any nonhomogeneous Dirichlet problem can be reduced to a homogeneous one by extending the boundary datum into the whole $\Omega_{f}$ (see, e.g., [24]).

2.2. The fluid-wall diffusion model. The previous model can be improved by considering the solute dynamics not only in the lumen, but also in the arterial wall (see, e.g., $[10,11]$ ). With this goal, assume that the computational domain is composed by the lumen $\Omega_{f} \subset \mathbb{R}^{d}(d=2,3)$ and by $\Omega_{w}$, representing the wall. The common interface between $\Omega_{f}$ and $\Omega_{w}$ is denoted by $\Gamma \subset \mathbb{R}^{d-1}, \Gamma \equiv \bar{\Omega}_{f} \cap \bar{\Omega}_{w}$ (see Figure 2.1). In what follows, we will denote $\Omega:=\overline{\Omega_{f} \cup \Omega_{w}}$ and $\partial \Omega$ its boundary.

Let us denote by $C_{f}(t, \mathbf{x})$ and $C_{w}(t, \mathbf{x})$ the concentrations of the solute in the blood and in the arterial wall, respectively. Observe that, in this case, the value of $C_{w}(t, \mathbf{x})$ on $\Gamma$ is no longer a given datum, rather it is another unknown of the problem.

We consider again the Navier-Stokes equations to describe the blood motion and an advection-diffusion equation for the solute to hold in the blood. Moreover, because of the very low velocity of the solvent inside the wall, we neglect the advection phenomena, so that $C_{w}(t, \mathbf{x})$ actually satisfies a pure diffusion equation (see [18]). Let $\mathbf{n}_{f}$ be the unit outward normal vector on $\Gamma$ with respect to $\Omega_{f}$ and $\mathbf{n}_{w}=-\mathbf{n}_{f}$. The dynamics of the solute in the vessel and in the arterial wall are expressed by the following problem, where $\mathbf{u}$ (and consequently $\boldsymbol{\sigma}(\mathbf{u}), \boldsymbol{\mu}_{f}(\mathbf{u})$ ) are provided by the solution of Problem 2.1.

Problem 2.3.

$$
\begin{aligned}
& \left\{\begin{array}{l}
\frac{\partial C_{f}}{\partial t}-\nabla \cdot\left(\boldsymbol{\mu}_{f} \nabla C_{f}\right)+\mathbf{u} \cdot \nabla C_{f}=f_{f} \quad \text { in } \quad \Omega_{f}, \quad t>0, \\
C_{f}=0 \quad \text { on } \quad \partial \Omega_{f} \backslash \Gamma, \quad t>0,
\end{array}\right. \\
& \left\{\begin{array}{l}
\frac{\partial C_{w}}{\partial t}-\nabla \cdot\left(\mu_{w} \nabla C_{w}\right)=f_{w} \quad \text { in } \quad \Omega_{w}, \quad t>0, \\
C_{w}=0 \quad \text { on } \quad \partial \Omega_{w} \backslash \Gamma, \quad t>0,
\end{array}\right.
\end{aligned}
$$

and we consider the following matching conditions at the interface:

$$
\begin{array}{r}
\mu_{w} \frac{\partial C_{w}}{\partial \mathbf{n}_{w}}=-\mathbf{n}_{f} \cdot\left(\boldsymbol{\mu}_{f} \nabla C_{f}\right) \quad \text { on } \quad \Gamma, \\
\mathbf{n}_{f} \cdot\left(\boldsymbol{\mu}_{f} \nabla C_{f}\right)+\zeta\left(C_{f}-C_{w}\right)=0 \quad \text { on } \quad \Gamma .
\end{array}
$$

$\boldsymbol{\mu}_{f}$ is again a function of the shear rate, according to (2.3), while $\mu_{w}$ can be assumed constant; $f_{f}$ and $f_{w}$ represent possible forcing terms for the solute dynamics (in the lumen and the wall, respectively). In particular, $f_{w}$ may take into account the consumption of the solute by the arterial tissue.

Equation (2.6) states the equality of the solute fluxes across $\Gamma$ and thus ensures the conservation of solute in the whole domain $\Omega$. Equation (2.7) is formally similar to $(2.2 \mathrm{~b})$; however, this time $C_{w}$ is unknown. Both (2.6) and (2.7) therefore provide a real coupling between the two values of the concentration $C_{f}$ and $C_{w}$. By virtue of 
(2.7), the interface condition (2.6) can be equivalently substituted by

$$
\mu_{w} \frac{\partial C_{w}}{\partial \mathbf{n}_{w}}+\zeta\left(C_{w}-C_{f}\right)=0 \quad \text { on } \quad \Gamma .
$$

The pair of conditions (2.7) and (2.8) is equivalent to the pair (2.6) and (2.7). However, when the problem is split into two subproblems (in the fluid and the wall, respectively) in view of its numerical study, the choice of one set of interface condition rather than the other leads to different substructuring iterative methods. Choosing the pair of conditions (2.7) and (2.8) yields a more quickly convergent iterative method, besides leading to a more elegant mathematical (weak) formulation of the problem.

\section{Mathematical analysis of the two models.}

3.1. Basic notation. The mathematical analysis of the wall-free as well as of the fluid-wall problems is based on the weak formulation of Problems 2.1, 2.2, and 2.3, both for the cases $d=2$ and $d=3$. In view of that, we need a few of basic notations.

$L^{p}$ and Sobolev spaces. In what follows, $L^{p}(\Omega)$ will denote the space of functions whose $p$ th power is integrable in $\Omega$. The norm in $L^{p}(\Omega)$ will be denoted by $\|\cdot\|_{L^{p}(\Omega}$. The scalar product in $L^{2}(\Omega)$ will be denoted with $(\cdot, \cdot)$ (both for scalar and vector functions). The space $L^{\infty}(\Omega)$ features the essentially bounded functions in $\Omega$, and for $s \in \mathbb{N}, H^{s}(\Omega)$ will denote the Sobolev space of functions $v \in L^{2}(\Omega)$ such that all their (distributional) derivatives of order up to $s$ are functions of $L^{2}(\Omega)$. Notice that $H^{0}(\Omega) \equiv L^{2}(\Omega)$. The norm in $H^{s}(\Omega)$ will be denoted by $\|\cdot\|_{H^{s}(\Omega)}$. Since in the subsequent analysis we will often have to distinguish between norms in $\Omega_{f}$ and $\Omega_{w}$, a subscript ( $f$ or $w$ ) will be added to the norm specification whenever needed.

Trace spaces. If $\Sigma \subset \partial \Omega$ is open and nonempty, then the trace space of $H^{s}(\Omega)$ $(s \geq 1)$, i.e., the space of functions defined on $\Sigma$ which are traces of functions belonging to $H^{s}(\Omega)$, is indicated by $H^{s-1 / 2}(\Sigma)$. We recall (see [16]) that the trace operator $\gamma: H^{s}(\Omega) \rightarrow H^{s-1 / 2}(\Sigma)$ is surjective and continuous and there exists an injective, linear, and continuous map $\mathcal{L}: H^{s-1 / 2}(\Sigma) \rightarrow H^{s}(\Omega)$ called lifting, such that $\lambda=\gamma \mathcal{L} \lambda$ for all $\lambda \in H^{s-1 / 2}(\Sigma)$. In particular, there exists a constant $\beta_{t}$ such that the following trace inequality holds:

$$
\|\gamma \phi\|_{H^{1 / 2}(\Sigma)} \leq \beta_{t}\|\phi\|_{H^{1}(\Omega)} \quad \forall \quad \phi \in H^{1}(\Omega) .
$$

In what follows, we will denote by $\gamma_{f}$ (resp., $\gamma_{w}$ ) the trace operator from $H^{1}\left(\Omega_{f}\right)$ (resp., $H^{1}\left(\Omega_{w}\right)$ ) to $H^{1 / 2}(\Gamma)$. Correspondingly, the lifting in $\Omega_{f}$ (resp., $\Omega_{w}$ ) of a function $\lambda \in H^{1 / 2}(\Gamma)$ will be denoted with $\mathcal{L}_{f} \lambda$ (resp., $\mathcal{L}_{w} \lambda$ ).

Let us denote with $H_{0}^{1}(\Omega)$ the subspace of $H^{1}(\Omega)$ made of functions whose trace vanishes on $\partial \Omega$ (see [16]). If the functions have null traces on a subset $\Sigma$ of the boundary with positive measure, we will denote the corresponding subspace of $H^{1}(\Omega)$ by $H_{\Sigma}^{1}(\Omega)$. In $H_{\Sigma}^{1}(\Omega)$ the following Poincaré inequality holds:

$$
\|\phi\|_{L^{2}(\Omega)} \leq \alpha\|\nabla \phi\|_{L^{2}(\Omega)} \quad \forall \quad \phi \in H_{\Sigma}^{1}(\Omega)
$$

$\alpha$ being a constant depending on $\Omega$. Owing to this inequality, the equivalence in $H_{\Sigma}^{1}(\Omega)$ between the $H^{1}$ norm of a function and the $L^{2}$ norm of its gradient holds true.

Now, suppose that $\Gamma$ denotes a $d-1$ dimensional manifold in $\bar{\Omega}\left(\subset \mathbb{R}^{d}\right)$ and consider the trace on $\Gamma$ of a function $u$ of $H_{\Sigma}^{1}(\Omega)$. If $\Gamma \cap \Sigma$ is nonempty, then the trace of $u$ on $\Gamma$ belongs to a subspace of $H^{1 / 2}(\Gamma)$ usually denoted by $H_{00}^{1 / 2}(\Gamma)$ (see [16]). 
Let $\rho$ and $\lambda$ be two functions of $H^{1 / 2}(\Sigma)$ and $\zeta$ be a positive function in $L^{2}(\Sigma)$. Then, the following definition makes sense:

$$
(\rho, \lambda)_{\zeta, \Sigma}=\int_{\Sigma} \zeta \rho \lambda d \gamma=(\sqrt{\zeta} \lambda, \sqrt{\zeta} \rho)
$$

Indeed, by Sobolev embedding of $H^{1 / 2}(\Sigma)$ in $L^{4}(\Sigma)$, the product $\lambda \rho$ belongs to $L^{2}(\Sigma)$. Denoting by $\mathcal{L} \lambda$ and $\mathcal{L} \rho$ any continuous lifting of $\lambda$ and $\rho$ from $\Sigma$ to $\Omega$, we obtain

$$
\left|(\rho, \lambda)_{\zeta, \Sigma}\right| \leq \beta_{e}^{2}\|\zeta\|_{L^{2}(\Sigma)}\|\lambda\|_{H^{1 / 2}(\Sigma)}\|\rho\|_{H^{1 / 2}(\Sigma)} \leq \beta^{2}\|\mathcal{L} \lambda\|_{H^{1}(\Omega)}\|\mathcal{L} \rho\|_{H^{1}(\Omega)},
$$

with $\beta^{2}=\beta_{e}^{2} \beta_{t}^{2}\|\zeta\|_{L^{2}(\Sigma)}$, where $\beta_{e}$ is the embedding constant of $H^{1 / 2}(\Sigma)$ in $L^{4}(\Sigma)$ and $\beta_{t}$ is the constant of the trace inequality (3.1). For the sake of simplification, we will drop the specification $\Sigma$ in $(\rho, \lambda)_{\zeta, \Sigma}$ since it will be clear from the context. Accordingly, we set

$$
\|\lambda\|_{\zeta}^{2}=(\lambda, \lambda)_{\zeta}=\|\sqrt{\zeta} \lambda\|^{2}
$$

Remark 3.1. If we suppose that $\zeta(\cdot)>0$ almost everywhere, $\|\lambda\|_{\zeta}$ is a norm of $\lambda$ equivalent to $\|\lambda\|_{H^{1 / 2}(\Sigma)}$. In the application at hand, this hypothesis implies that the vascular tissue has a minimal nonzero permeability independently of the value of the shear stress on the wall. Although being realistic (see [19]), we will not make this assumption in our analysis any further.

Space-time functions. When considering space-time functions $v:(0, T) \times \Omega \rightarrow \mathbb{R}$, we introduce the space

$$
L^{2}\left(0, T ; H^{s}(\Omega)\right) \equiv\left\{v:(0, T) \rightarrow H^{s} \mid v(t) \text { is measurable, } \int_{0}^{T}\|v(t)\|_{H^{s}(\Omega)}^{2} d t<\infty\right\}
$$

endowed with the norm

$$
\|v\|_{L^{2}\left(0, T ; H^{s}(\Omega)\right)} \equiv\left(\int_{0}^{T}\|v(t)\|_{H^{s}(\Omega)}^{2} d t\right)^{1 / 2}
$$

We then set

$$
\begin{aligned}
L^{\infty}\left(0, T ; L^{2}(\Omega)\right) \equiv & \left\{v:(0, T) \rightarrow L^{2} \mid v(t)\right. \text { is measurable, } \\
& \left.\quad \text { and }\|v(t, \cdot)\|_{L^{2}(\Omega)}^{2} \text { is essentially bounded in }(0, T)\right\}
\end{aligned}
$$

endowed with the norm

$$
\|v\|_{L^{\infty}\left(0, T ; L^{2}(\Omega)\right)} \equiv \inf \left\{M>0 \mid\|v(t, \cdot)\|_{L^{2}(\Omega)}^{2} \leq M \text { almost everywhere in }(0, T)\right\} .
$$

3.2. The Navier-Stokes problem. As pointed out in the introduction, blood is essentially an incompressible fluid, which can be mathematically described by means of the classical Navier-Stokes equations. Since the velocity field of blood influences the solutes dynamics, its regularity affects the analysis of the solutes problem. It is therefore worthwhile recalling some results about the solution of the Navier-Stokes equations.

The weak formulation of the Navier-Stokes problem reads as follows. 
Problem 3.1. Given $\mathbf{u}_{0} \in\left(L^{2}\left(\Omega_{f}\right)\right)^{d}$ with $\nabla \cdot \mathbf{u}_{0}=0$ and $\mathbf{f} \in L^{2}\left(0, T ;\left(L^{2}\left(\Omega_{f}\right)\right)^{d}\right)$, find $\mathbf{u} \in L^{2}\left(0, T ;\left(H^{1}\left(\Omega_{f}\right)\right)^{d}\right) \cap L^{\infty}\left(0, T ;\left(L^{2}\left(\Omega_{f}\right)\right)^{d}\right)$ and $P \in L^{2}\left(0, T ; L^{2}\left(\Omega_{f}\right)\right)$ such that for all $t>0$

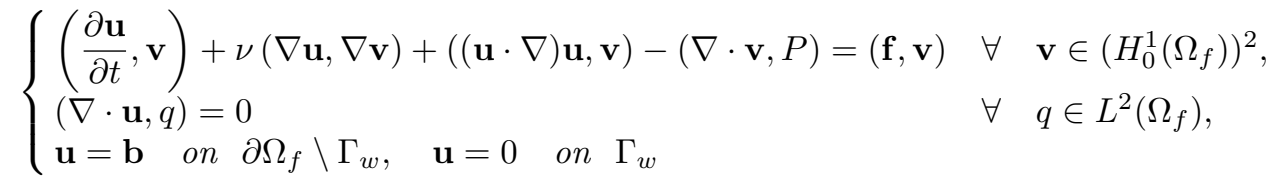

with $\mathbf{u}(0)=\mathbf{u}_{0}$ for $t=0$.

We recall that existence of the solution has been proved by Leray $[13,15,14]$ and Hopf [7]; the uniqueness has been proved in the two-dimensional case, while in the three-dimensional case it is still an open problem (see, e.g., $[28,29]$ ). Moreover, it is possible to prove that the solution is regular when the boundary $\partial \Omega_{f}$ and the initial datum $\mathbf{u}_{0}$ are more regular. In particular, we recall the following results that will be particularly useful to our purpose (its proof can be found in [6]).

Theorem 3.1. Let $\mathbf{u}_{0} \in H^{2}\left(\Omega_{f}\right)$ and $\mathbf{f}$ be smooth enough (e.g., $\mathbf{f} \in L^{\infty}\left(\Omega_{f}\right)$ and $\left.\nabla \mathbf{f} \in L^{\infty}\left(\Omega_{f}\right)\right)$. Assume that

- (Cattabriga assumption on the domain $\Omega_{f}$ ) for a given $\mathbf{g} \in \mathbf{L}^{2}\left(\Omega_{f}\right)$, the steady Stokes problem

$$
-\Delta \mathbf{v}+\nabla q=\mathbf{g}, \quad \nabla \cdot \mathbf{v}=0 \quad \text { in } \Omega_{f},\left.\quad \mathbf{v}\right|_{\partial \Omega}=\mathbf{0},
$$

has a unique solution that satisfies the inequality

$$
\|\mathbf{v}\|_{H^{2}\left(\Omega_{f}\right)}+\|q\|_{H^{1}\left(\Omega_{f}\right) \backslash \mathbb{R}} \leq c\|\mathbf{g}\|_{L^{2}\left(\Omega_{f}\right)},
$$

where $c$ is a constant;

- there exists a time $T, 0<T \leq \infty$ and a constant $A$ such that the solution of the Navier-Stokes problem (3.5) satisfies

$$
\sup _{0<t<T}\|\nabla \mathbf{u}(\cdot, t)\|_{L^{2}\left(\Omega_{f}\right)} \leq A .
$$

Then there exists a constant B such that the solution of the Navier-Stokes problem (3.5) satisfies

$$
\sup _{0<t<T}\|\mathbf{u}(\cdot, t)\|_{H^{2}\left(\Omega_{f}\right)} \leq B .
$$

Whenever we consider the dependence of the diffusivity tensor $\boldsymbol{\mu}_{f}$ on the shear rate (i.e., $\mathcal{D} \neq 0$ in (2.3)), the following result is useful (for its proof, see [5, Theorem 9]).

TheOrem 3.2. Suppose that the hypotheses of the previous theorem hold. Moreover, let $\Omega$ be any three-dimensional domain whose boundary is uniformly of class $C^{3}$. Suppose that the boundary value prescribed can be extended to a solenoidal function $\mathbf{g}$ smooth enough and that $\mathbf{f} \in C^{\infty}((0, \infty) \times \bar{\Omega})$. Then, on some interval $[0, T]$, there exists a solution $\mathbf{u}, P$ of the Navier-Stokes problem such that, in particular, $\mathbf{u} \in C([0, T) \times \bar{\Omega}) \cap C^{\infty}((0, T) \times \Omega)$.

Should the assumptions of Theorem 3.2 hold, we conclude that $\boldsymbol{\mu}_{f}$ is a bounded vector function, i.e.,

$$
\left|\boldsymbol{\mu}_{f}(t, \mathbf{x})\right| \leq m \quad \forall \quad \mathbf{x} \in \Omega, t>0,
$$

where $m$ is a suitable constant. 
3.3. The wall-free model. Let us introduce the bilinear form

$$
a_{f}\left(\psi_{f}, \phi_{f}\right)=\left(\boldsymbol{\mu}_{f} \nabla \psi_{f}, \nabla \phi_{f}\right)+\left((\mathbf{u} \cdot \nabla) \psi_{f}, \phi_{f}\right)
$$

with $\phi_{f}, \psi_{f} \in H^{1}\left(\Omega_{f}\right)$. Observe that property (3.9) and Sobolev embedding theorems (see, e.g., [24]) ensure that for all $\phi_{f}$ and $\psi_{f}, a_{f}(\cdot, \cdot)$ is well defined and continuous.

Indeed,

$$
\begin{aligned}
\left|a_{f}\left(\psi_{f}, \phi_{f}\right)\right| \leq & m\left\|\nabla \psi_{f}\right\|_{L^{2}\left(\Omega_{f}\right)}\left\|\nabla \phi_{f}\right\|_{L^{2}\left(\Omega_{f}\right)} \\
& +\|\mathbf{u}\|_{L^{4}\left(\Omega_{f}\right)}\left\|\phi_{f}\right\|_{L^{4}\left(\Omega_{f}\right)}\left\|\nabla \psi_{f}\right\|_{L^{2}\left(\Omega_{f}\right)} \\
\leq & \left(m+\|\mathbf{u}\|_{H^{1}\left(\Omega_{f}\right)}\right)\left\|\psi_{f}\right\|_{H^{1}\left(\Omega_{f}\right)}\left\|\phi_{f}\right\|_{H^{1}\left(\Omega_{f}\right)}
\end{aligned}
$$

The weak formulation of (2.2) reads as follows.

Problem 3.2. Let $C_{f, 0} \in L^{2}\left(\Omega_{f}\right)$ and $\kappa_{w}(t) \in H^{1 / 2}\left(\Gamma_{w}\right)$ (for all $t>0$ ) be two given functions and let $\mathbf{u}(t, \mathbf{x})$ be the solution of Problem 3.1. Then, we look for a function $C_{f} \in L^{2}\left(0, T ; H_{\partial \Omega_{f} \backslash \Gamma_{w}}^{1}\left(\Omega_{f}\right)\right) \cap L^{\infty}\left(0, T ; L^{2}\left(\Omega_{f}\right)\right)$ which satisfies for all $t>0$

$$
\left(\frac{\partial C_{f}}{\partial t}, \phi\right)+a_{f}\left(C_{f}, \phi\right)+\left(C_{f}, \phi\right)_{\zeta}=\left(\kappa_{w}, \phi\right)_{\zeta}+\left(f_{f}, \phi\right)
$$

for all $\phi \in H_{\partial \Omega \backslash \Gamma_{w}}^{1}\left(\Omega_{f}\right)$, with $C_{f}=C_{f, 0}$ at $t=0$.

The following result holds.

Lemma 3.1. If the solution $\mathbf{u}$ of Problem 3.1 is smooth enough-namely, according to the features given in Theorem 3.2-then Problem 3.2 admits a unique solution, which depends continuously on the data.

Proof. Owing to (3.4) and the regularity of $\mathbf{u}$, the right-hand side in (3.12) is a linear and continuous functional in $H_{\partial \Omega \backslash \Gamma_{w}}^{1}\left(\Omega_{f}\right)$ and the bilinear form $a_{f}(\psi, \phi)+$ $(\psi, \phi)_{\zeta}$ is continuous for all $\phi, \psi \in H^{1}\left(\Omega_{f}\right)$. Moreover, this bilinear form is coercive. Indeed, since $\nabla \cdot \mathbf{u}=0$ and $\left.\mathbf{u}\right|_{\Gamma_{w}}=0$, owing to (2.3), we have

$$
a_{f}(\psi, \psi)+(\psi, \psi)_{\zeta} \geq \mu_{f 0}\|\nabla \psi\|_{L^{2}\left(\Omega_{f}\right)}^{2}+\int_{\Gamma_{w}} \zeta \psi^{2} d \gamma \quad \forall \psi \in H_{\partial \Omega_{f} \backslash \Gamma_{w}}^{1}\left(\Omega_{f}\right) .
$$

Since $\zeta \geq 0$, by virtue of the Poincaré inequality there exists a positive constant $\alpha_{f}$ such that

$$
a_{f}(\psi, \psi) \geq \alpha_{f}\|\psi\|_{H^{1}\left(\Omega_{f}\right)}^{2} .
$$

By standard arguments about parabolic problems (see, e.g., [24]), the continuity of the right-hand side in (3.12) together with continuity and coercivity of $a_{f}(\cdot, \cdot)$ allows us to conclude that Problem 3.2 has a unique solution, depending continuously on the data $\kappa_{w}$ and $f_{f}$.

Remark 3.2. Obviously, for constitutive laws for the diffusivity $\boldsymbol{\mu}_{f}$ different from the one in (2.3), or for specific values of the parameters ( $\epsilon$ in particular), less regularity to the velocity field (with respect to the specifications of Theorem 3.2) can be required in order for $a_{f}(\cdot, \cdot)$ to be well defined and continuous. In particular, if we assume that the diffusivity of the solute does not depend on the shear rate (i.e., we set $\mathcal{D}=0$ in (2.3)), it is enough that $\mathbf{u} \in H^{3 / 2}\left(\Omega_{f}\right)$ in order to make the term $(\cdot, \cdot)_{\zeta}$ well defined and depending continuously on its arguments. Indeed, if $\mathbf{u} \in H^{3 / 2}\left(\Omega_{f}\right)$ for every $t>0$, then $\boldsymbol{\sigma}(\mathbf{u}) \in L^{2}\left(\Gamma_{w}\right)$. Moreover, by hypothesis, both $C_{f}$ and $\kappa_{w}$ belong to $H^{1 / 2}\left(\Gamma_{w}\right)$ and henceforth to $L^{4}\left(\Gamma_{w}\right)$; therefore $\left(C_{f}, \phi\right)_{\zeta}$ and $\left(\kappa_{w}, \phi\right)_{\zeta}$ are finite for all $\phi \in H^{1}\left(\Omega_{f}\right)$.

Finally, the advective term $\left((\mathbf{u} \cdot \nabla) C_{f}, \phi\right)$ is bounded, as $\mathbf{u} \in H^{1}(\Omega)$. 
3.4. The fluid-wall model. The weak formulation of the coupled Problem 2.3 is obtained as follows.

Let us consider the weak formulation of the "fluid-side" problem, which can be obtained by proceeding exactly as for Problem 3.2, leading to the equation

$$
\left(\frac{\partial C_{f}}{\partial t}, \phi_{f}\right)+a_{f}\left(C_{f}, \phi_{f}\right)+\left(C_{f}-C_{w}, \phi_{f}\right)_{\zeta}=\left(f_{f}, \phi_{f}\right)
$$

for all $\phi_{f} \in H_{\partial \Omega_{f} \backslash \Gamma}^{1}\left(\Omega_{f}\right)$, with $C_{f}=C_{f, 0}$ for $t=0$. This time, the interface integrals $(\cdot, \cdot)_{\zeta}$ are carried out on the fluid-wall interface $\Gamma$.

Proceeding in the same way as for the "wall-side" problem, i.e., by multiplying equation (2.5) by a test function $\phi_{w} \in H_{\partial \Omega_{w} \backslash \Gamma}^{1}\left(\Omega_{w}\right)$, integrating in $\Omega_{w}$, and then applying the Green formula, we obtain the weak formulation of the wall problem.

More precisely, similarly to (3.10), for all $\phi_{w}, \psi_{w} \in H^{1}\left(\Omega_{w}\right)$, we set

$$
a_{w}\left(\psi_{w}, \phi_{w}\right)=\mu_{w}\left(\nabla \psi_{w}, \nabla \phi_{w}\right) .
$$

Observe that the bilinear form $a_{w}(\cdot, \cdot)$ is continuous, coercive and, in particular, symmetric, i.e.,

$$
a_{w}\left(\psi_{w}, \phi_{w}\right)=a_{w}\left(\phi_{w}, \psi_{w}\right)
$$

for all $\psi_{w}, \phi_{w} \in H^{1}\left(\Omega_{w}\right)$. Now, referring to boundary conditions (2.7) and (2.8), the weak formulation of the wall-side equation reads as

$$
\left(\frac{\partial C_{w}}{\partial t}, \phi_{w}\right)+a_{w}\left(C_{w}, \phi_{w}\right)+\left(C_{w}-C_{f}, \phi_{w}\right)_{\zeta}=\left(f_{w}, \phi_{w}\right)
$$

for all $\phi_{w} \in H_{\partial \Omega_{w} \backslash \Gamma}^{1}\left(\Omega_{w}\right)$ with the initial condition $C_{w}=C_{w, 0}$ for $t=0$. Observe that (3.15) and (3.17) are formally very similar, provided each equation is considered in the respective domain.

In order to give a compact vector formulation of the coupled problem (3.15), (3.17), let us define the product space

$$
\mathbf{H}:=H_{\partial \Omega_{f} \backslash \Gamma}^{1}\left(\Omega_{f}\right) \times H_{\partial \Omega_{w} \backslash \Gamma}^{1}\left(\Omega_{w}\right)
$$

endowed with the norm

$$
\|\boldsymbol{\Phi}\|_{\mathbf{H}} \equiv\left(\left\|\phi_{f}\right\|_{H^{1}\left(\Omega_{f}\right)}^{2}+\left\|\phi_{w}\right\|_{H^{1}\left(\Omega_{w}\right)}^{2}\right)^{1 / 2} .
$$

Let us define a vector unknown $\mathbf{C} \equiv\left[C_{f}, C_{w}\right]$, a forcing vector $\mathbf{F}=\left[f_{f}, f_{w}\right]$ and a vector test function $\boldsymbol{\Phi}=\left[\phi_{f}, \phi_{w}\right] \in \mathbf{H}$. Summing up (3.15) and (3.17), we obtain the following problem for the unknown $\mathbf{C}$.

Problem 3.3. Given $\left[C_{f, 0}, C_{w, 0}\right] \in L^{2}(\Omega)$ and $\mathbf{F} \in L^{2}\left(0, T, L^{2}(\Omega)\right)$, for all $t>0$ find $\mathbf{C} \in L^{2}(0, T ; \mathbf{H}) \cap L^{\infty}\left(0, T ; L^{2}(\Omega)\right)$ such that

$$
\left(\frac{\partial \mathbf{C}}{\partial t}, \boldsymbol{\Phi}\right)+\mathcal{A}(\mathbf{C}, \boldsymbol{\Phi})=(\mathbf{F}, \boldsymbol{\Phi}) \quad \forall \quad \boldsymbol{\Phi} \in \mathbf{H}
$$

with $\mathbf{C}(0)=\left(C_{f, 0}, C_{w, 0}\right)$ for $t=0$, where

$$
\mathcal{A}(\mathbf{C}, \boldsymbol{\Phi}):=a_{f}\left(C_{f}, \phi_{f}\right)+a_{w}\left(C_{w}, \phi_{w}\right)+\left(C_{f}-C_{w}, \phi_{f}-\phi_{w}\right)_{\zeta}
$$


is the bilinear form associated to the coupled Problem 3.3.

The wellposedness of this problem is proven in the following theorem.

TheOREM 3.3. Under the hypotheses of Theorem 3.2, Problem 3.3 admits a unique solution which depends continuously on the data.

Proof. Proceeding exactly as in the proof of Lemma 3.1, we see that the righthand side of (3.18) is a linear and continuous functional in $\mathbf{H}$, and the regularity of $\mathbf{u}$ ensures that the bilinear form $\mathcal{A}(\mathbf{C}, \boldsymbol{\Phi})$ is continuous in $\mathbf{H}$ for every $t>0$. Moreover, $\mathcal{A}(\mathbf{C}, \boldsymbol{\Phi})$ is coercive. This follows from the coercivity of $a_{f}(\cdot, \cdot)$ and $a_{w}(\cdot, \cdot)$. Indeed, there exists a constant $\alpha>0$ such that, for all $\mathbf{\Phi} \in \mathbf{H}$,

$$
\mathcal{A}(\boldsymbol{\Phi}, \boldsymbol{\Phi})=a_{f}\left(\phi_{f}, \phi_{f}\right)+a_{w}\left(\phi_{w}, \phi_{w}\right)+\left\|\phi_{f}-\phi_{w}\right\|_{\zeta}^{2} \geq \alpha\|\boldsymbol{\Phi}\|_{\mathbf{H}}^{2} .
$$

In fact, the coercivity of $a_{f}(\cdot, \cdot)$ has been proved in (3.14). On the other hand the coercivity of $a_{w}(\cdot, \cdot)$ is again an immediate consequence of the Poincaré inequality.

The wellposedness of Problem 3.3 is therefore proven by a standard application of the Faedo-Galerkin method (see, e.g., [24]).

Remark 3.3. Let us point out again that, if the diffusivity of the solute in fluid does not depend on the shear rate, the smoothness requirement on $\mathbf{u}$ can be relaxed, exactly as done in Remark 3.2.

\section{Numerical approximation.}

4.1. Time and space discretization of Problems 3.2 and 3.3. Both Problems 3.2 and 3.3 involve the coupling of the Navier-Stokes equations (Problem 3.1) with an advection-diffusion problem. In our models, the advection-diffusion equation depends on the Navier-Stokes solution, through the advective field, the boundary permeability, and possibly the augmented diffusivity. Hence the Navier-Stokes problem is solved at a first step, and then, with the vector field $\mathbf{u}$, the shear rate $\mathbf{d}$, and the shear stress $\boldsymbol{\sigma}(\mathbf{u})$ available, we solve the advection-diffusion problem.

For the space discretization of the equations at hand, we use the finite element method. In particular, for what concerns the Navier-Stokes equations, in order to satisfy the compatibility inf-sup condition, we have adopted a linear approximation based on the so-called $\mathbb{P}^{1}$ iso $\mathbb{P}^{2}-\mathbb{P}^{1}$ element, while the backward Euler time discretization has been coupled with a semi-implicit treatment of the nonlinear term. Finally, a splitting of the velocity and pressure problem based on the so-called Yosida method is carried out. For more details about these techniques, the interested reader is referred to $[24,20,21]$.

Referring to the advection-diffusion equations, both Problems 3.2 and 3.3 feature a very low diffusivity of the solute. In other terms, these problems are dominated by advection effects. Indeed, if $h$ denotes the space discretization step (which in our simulation is equal to $\left.10^{-2} \mathrm{~cm}\right),|\mathbf{u}|$ is a representative value of the blood velocity, for instance equal to $10 \mathrm{~cm} / \mathrm{s}$, and the diffusivity is equal to $\zeta=10^{-5} \mathrm{~cm}^{2} / \mathrm{s}$, we have an indicative value of the Péclet number (which weighs the convection effects with respect to the diffusive ones) of $10^{4}$. As it is well known, finite element techniques (and in general Galerkin methods) could be inaccurate when facing convection dominated problems and resorting to a stabilization technique becomes mandatory. Different strategies can be pursued in this regard: the interested reader is referred to [8] and [24]. In our simulations, streamline-upwind/Petrov-Galerkin (SUPG) has been successfully adopted and will be addressed later on.

4.2. An iterative subdomains scheme for the fluid-wall model. In this section, we focus our attention on the numerical solution of Problem 3.3. This problem 
deals indeed with two differential subproblems to be solved in different parts of the computational domain. For the sake of numerical efficiency, we suitably split the whole problem into a sequence of two subproblems in the two physical subdomains (namely, the lumen and the arterial wall), to be solved in an iterative framework. This approach can be therefore seen in the general framework of the iterative substructuring methods (see [25]).

Different splitting strategies can be adopted according to different choices for the interface conditions prescribed to each subproblem as boundary conditions. As previously pointed out, our choice is to consider the interface conditions (2.7) and (2.8).

In particular, here we introduce the adopted splitting scheme, analyzing in detail the features of the subproblems to be solved at each step. In section 4.3, we will prove the convergence of the solution computed by the iterative scheme to the solution of the coupled lumen-wall problem.

First of all, let us introduce the time discretization of Problem 3.3, subdividing the time interval $[0, T]$ in $N$ time steps $t^{n}=n \Delta t$, with $\Delta t>0$ and $n=1, \ldots, N$, and use the backward Euler finite difference scheme for the time differential problem. Then, set $\chi=1 / \Delta t$ and

$$
\begin{aligned}
\widehat{a}_{f}\left(\psi_{f}, \phi_{f}\right) & =\chi\left(\psi_{f}, \phi_{f}\right)+a_{f}\left(\psi_{f}, \phi_{f}\right), \\
\widehat{a}_{w}\left(\psi_{w}, \phi_{w}\right) & =\chi\left(\psi_{w}, \phi_{w}\right)+a_{w}\left(\psi_{w}, \phi_{w}\right) .
\end{aligned}
$$

As for $a_{w}(\cdot, \cdot)$, observe that $\widehat{a}_{w}(\cdot, \cdot)$ is symmetric. With this notation, the timediscrete (continuous-in-space) counterpart of Problem 3.3 can be formulated as follows.

Problem 4.1. Given $C_{f}^{0}=C_{f, 0} \in L^{2}\left(\Omega_{f}\right)$ and $C_{w}^{0}=C_{w, 0} \in L^{2}\left(\Omega_{w}\right)$, for $n=0,1, \ldots, N$ find $\left[C_{f}^{n+1}, C_{w}^{n+1}\right] \in \mathbf{H}$ such that for all $\phi_{f} \in H_{\partial \Omega_{f} \backslash \Gamma}^{1}\left(\Omega_{f}\right)$ and $\phi_{w} \in$ $H_{\partial \Omega_{w} \backslash \Gamma}^{1}\left(\Omega_{w}\right)$,

$\widehat{a}_{f}\left(C_{f}^{n+1}, \phi_{f}\right)+\left(C_{f}^{n+1}-C_{w}^{n+1}, \phi_{f}\right)_{\zeta}=\chi\left(C_{f}^{n}, \phi_{f}\right)+\left(f_{f}^{n+1}, \phi_{f}\right) \phi_{f} \in H_{\partial \Omega_{f} \backslash \Gamma}^{1}\left(\Omega_{f}\right)$,

$\widehat{a}_{w}\left(C_{w}^{n+1}, \phi_{w}\right)+\left(C_{w}^{n+1}-C_{f}^{n+1}, \phi_{w}\right)_{\zeta}=\chi\left(C_{w}^{n}, \phi_{w}\right)+\left(f_{w}^{n+1}, \phi_{w}\right) \phi_{w} \in H_{\partial \Omega_{w} \backslash \Gamma}^{1}\left(\Omega_{w}\right)$,

where $f_{f}^{n}$ and $f_{w}^{n}$ denote the value of the functions $f_{f}$ and $f_{w}$ at time $t^{n}$.

Remark 4.1. The bilinear forms $\widehat{a}_{f}(\cdot, \cdot), \widehat{a}_{w}(\cdot, \cdot)$ are continuous and coercive, with coercivity constants $\widehat{\alpha}_{f}$ and $\widehat{\alpha}_{w}$, which are related to the Poincaré inequality constants in $\Omega_{f}$ and $\Omega_{w}$, respectively. Therefore, proceeding as for Problem 3.3, it can be proven that the time discrete Problem 4.1 is well posed.

The splitting of Problem 4.1 into subproblems, one in the fluid domain $\Omega_{f}$, the other in $\Omega_{w}$, can be carried out as follows.

To start the iterative method, an initial guess for $C_{w}^{n+1}$ is needed; it will be denoted by $C_{w, 0}^{n+1}$. Then, we find the sequence of functions $\left[C_{f, k}^{n+1}, C_{w, k}^{n+1}\right] \in \mathbf{H}$, by solving the following equations for $k=0,1, \ldots$ :

$$
\begin{aligned}
& \widehat{a}_{f}\left(C_{f, k+1}^{n+1}, \phi_{f}\right)+\left(C_{f, k+1}^{n+1}, \phi_{f}\right)_{\zeta} \\
& \quad=\chi\left(C_{f}^{n}, \phi_{f}\right)+\left(C_{w, k}^{n+1}, \phi_{f}\right)_{\zeta}+\left(f_{f}^{n+1}, \phi_{f}\right) \quad \forall \quad \phi_{f} \in H_{\partial \Omega_{f} \backslash \Gamma}^{1}\left(\Omega_{f}\right),
\end{aligned}
$$


and

$$
\begin{aligned}
& \widehat{a}_{w}\left(C_{w, k+1}^{n+1}, \phi_{w}\right)+\left(C_{w, k+1}^{n+1}, \phi_{w}\right)_{\zeta} \\
& \quad=\chi\left(C_{w}^{n}, \phi_{w}\right)+\left(C_{f, k+1}^{n+1}, \phi_{w}\right)_{\zeta}+\left(f_{w}^{n+1}, \phi_{w}\right) \quad \forall \quad \phi_{w} \in H_{\partial \Omega_{w} \backslash \Gamma}^{1}\left(\Omega_{w}\right) .
\end{aligned}
$$

4.3. Convergence analysis of the substructuring iterative method. The analysis of convergence for the splitting method (4.5)-(4.6) does not straightforwardly follow from available convergence results, due to the lack of continuity between $C_{f, k+1}^{n+1}$ and $C_{w, k+1}^{n+1}$ at the subdomain interface $\Gamma$. In the subsequent analysis, we will focus our attention on the convergence of the sequence $\left[C_{f, k}^{n+1}, C_{w, k}^{n+1}\right]$ to the solution $\left[C_{f}^{n+1}, C_{w}^{n+1}\right]$ at the time step $n+1$. Since all the relevant equations for the convergence analysis will deal only with quantities evaluated at the time level $n+1$, for notational convenience we will drop the index identifying the time level. Thus, we will use $C_{f, k}$ instead of $C_{f, k}^{n+1}$ and $C_{w, k}$ instead of $C_{w, k}^{n+1}$. The time index will be explicitly indicated only when referring to a different time step.

Finally, let us introduce the splitting error:

$$
e_{f, k}:=C_{f}-C_{f, k}, \quad e_{w, k}:=C_{w}-C_{w, k}, \quad \lambda_{k}:=\gamma_{w} e_{w, k}
$$

where $\left[C_{f}, C_{w}\right]$ is the solution of Problem 4.1. From (4.7) it follows that $e_{f, k} \in$ $H_{\partial \Omega_{f} \backslash \Gamma}^{1}\left(\Omega_{f}\right), e_{w, k} \in H_{\partial \Omega_{w} \backslash \Gamma}^{1}\left(\Omega_{w}\right)$, and $\lambda_{k} \in H^{1 / 2}(\Gamma)$. More precisely, since $\Gamma \cap \partial \Omega \neq \emptyset$, and the concentration is null on $\partial \Omega$, the trace $\lambda_{k}$ belongs to $H_{00}^{1 / 2}(\Gamma)$, according to the definition given in section 3.1. In what follows, we will set $\Lambda:=H_{00}^{1 / 2}(\Gamma)$.

Now if we subtract memberwise equations (4.3) and (4.5) and correspondingly (4.4) and (4.6), given an initial guess $\lambda_{0}$, we obtain, for all $k=0,1, \ldots$,

$$
\begin{aligned}
\widehat{a}_{f}\left(e_{f, k+1}, \phi_{f}\right)+\left(e_{f, k+1}, \phi_{f}\right)_{\zeta} & =\left(\lambda_{k}, \phi_{f}\right)_{\zeta} \quad \forall \quad \phi_{f} \in H_{\partial \Omega_{f} \backslash \Gamma}^{1}\left(\Omega_{f}\right), \\
\widehat{a}_{w}\left(e_{w, k+1}, \phi_{w}\right)+\left(e_{w, k+1}, \phi_{w}\right)_{\zeta} & =\left(\gamma_{f} e_{f, k+1}, \phi_{w}\right)_{\zeta} \quad \forall \quad \phi_{w} \in H_{\partial \Omega_{w} \backslash \Gamma}^{1}\left(\Omega_{w}\right) .
\end{aligned}
$$

We will refer to (4.8) and (4.9) as splitting error equations.

Let us introduce the following definitions.

- Let $\mathcal{R}_{f}: \Lambda \rightarrow \Lambda$ be the following operator. For a given function $\psi \in \Lambda$, $\mathcal{R}_{f} \psi=\gamma_{f} u_{f}$, where $u_{f} \in H_{\partial \Omega_{f} \backslash \Gamma}^{1}\left(\Omega_{f}\right)$ satisfies

$$
\widehat{a}_{f}\left(u_{f}, \phi_{f}\right)+\left(u_{f}, \phi_{f}\right)_{\zeta}=\left(\psi, \phi_{f}\right)_{\zeta} \quad \forall \quad \phi_{f} \in H_{\partial \Omega_{f} \backslash \Gamma}^{1}\left(\Omega_{f}\right) .
$$

- Let $\mathcal{R}_{w}: \Lambda \rightarrow \Lambda$ be the following operator. For a given function $\xi \in \Lambda$, $\mathcal{R}_{w} \xi=\gamma_{w} u_{w}$, where $u_{w} \in H_{\partial \Omega_{w} \backslash \Gamma}^{1}\left(\Omega_{w}\right)$ satisfies

$$
\widehat{a}_{w}\left(u_{w}, \phi_{w}\right)+\left(u_{w}, \phi_{w}\right)_{\zeta}=\left(\xi, \phi_{w}\right)_{\zeta} \quad \forall \quad \phi_{w} \in H_{\partial \Omega_{w} \backslash \Gamma}^{1}\left(\Omega_{w}\right) .
$$

The iterative scheme corresponding to the error equations (4.8), (4.9) can now be regarded as a fixed point iteration:

$$
\lambda_{k+1}=T \lambda_{k}, \quad k=0,1, \ldots, \quad \text { where } \quad T=\mathcal{R}_{w} \cdot \mathcal{R}_{f} .
$$

In order to prove the convergence of the scheme, we prove the following preliminary result. 
Lemma 4.1. There exists a constant $K<1$ depending on $\Omega_{f}, \Omega_{w}$, and $\zeta$ such that

$$
\|T \psi\|_{\zeta} \leq K\|\psi\|_{\zeta} \quad \forall \quad \psi \in \Lambda .
$$

Proof. Let $u_{f}$ be the function associated to $\psi$ through problem (4.10). We set $\xi=\mathcal{R}_{f} \psi$ so that $\xi=\gamma_{f} u_{f}$. Set, moreover, $\lambda=\mathcal{R}_{w} \xi$, so that $\lambda=T \psi$. There exists a constant $K_{f}<1$, depending on $\Omega_{f}$ and $\zeta$, such that

$$
\|\xi\|_{\zeta} \leq K_{f}\|\psi\|_{\zeta} \quad \forall \quad \psi \in \Lambda .
$$

Indeed, by choosing $\phi_{f}=u_{f}$ in (4.10), we obtain

$$
\widehat{\alpha}_{f}\left\|u_{f}\right\|_{H^{1}\left(\Omega_{f}\right)}^{2}+\|\xi\|_{\zeta}^{2} \leq\|\psi\|_{\zeta}\|\xi\|_{\zeta} .
$$

From (3.4), we have that for all $\xi \in \Lambda$

$$
\|\xi\|_{\zeta}^{2} \leq K_{2}\left\|u_{f}\right\|_{H^{1}\left(\Omega_{f}\right)}^{2},
$$

with $K_{2}=\beta_{e}^{2} \beta_{t, f}^{2}\|\zeta\|_{L^{2}(\Gamma)}^{2}, \beta_{e}$ being the embedding constant of $\Lambda$ in $L^{4}(\Gamma)$ and $\beta_{t, f}$ the trace constant of inequality (3.1). Therefore, (4.15) yields

$$
\left(\frac{\widehat{\alpha}_{f}}{K_{2}}+1\right)\|\xi\|_{\zeta} \leq\|\psi\|_{\zeta}
$$

that is, inequality (4.14) with $K_{f}=\frac{K_{2}}{K_{2}+\widehat{\alpha}_{f}}<1$.

In a similar way, we can prove that there exists a constant $K_{w}<1$ such that

$$
\|\lambda\|_{\zeta} \leq K_{w}\|\xi\|_{\zeta} \quad \forall \quad \xi \in \Lambda
$$

Indeed, from (4.11) with $\phi_{w}=\lambda$, being $\lambda=\gamma_{w} u_{w}$, we obtain

$$
\widehat{\alpha}_{w}\left\|u_{w}\right\|_{H^{1}\left(\Omega_{w}\right)}^{2}+\|\lambda\|_{\zeta}^{2} \leq\|\xi\|_{\zeta}\|\lambda\|_{\zeta},
$$

and proceeding as for (4.17), we obtain (4.18) with $K_{w}=\frac{K_{4}}{K_{4}+\widehat{\alpha}_{w}}$, where (with obvious meaning of the notation) $K_{4}=\beta_{e}^{2} \beta_{t, w}^{2}\|\zeta\|_{L^{2}(\Gamma)}^{2}$.

Now, assembling (4.14) and (4.18), we have

$$
\|\lambda\|_{\zeta} \leq \frac{K_{4}}{K_{4}+\widehat{\alpha}_{w}} \frac{K_{2}}{K_{2}+\widehat{\alpha}_{f}}\|\psi\|_{\zeta}<\|\psi\|_{\zeta}
$$

which concludes the proof.

As an immediate consequence of Lemma 4.1, we have

$$
\lim _{k \rightarrow \infty}\left\|\lambda_{k}\right\|_{\zeta}=0
$$

Remark 4.2. If we suppose that $\zeta>0$ a.e. on $\Gamma$ (see Remark 3.1), Lemma 4.1 states that $T$ is a contraction in $\Lambda$ with respect to the metric induced by the norm $\|\cdot\|_{\zeta}$. Correspondingly, (4.21) yields $\left\|e_{w, k}\right\|_{\Lambda}$ tends to zero as $k \rightarrow \infty$.

Now, we can prove the following convergence result.

COROLlaRY 4.1. The scheme (4.5), (4.6) is convergent, precisely

$$
\lim _{k \rightarrow \infty}\left(\left\|e_{f, k}\right\|_{H^{1}\left(\Omega_{f}\right)}+\left\|e_{w, k}\right\|_{H^{1}\left(\Omega_{w}\right)}\right)=0 .
$$


Proof. The thesis is a consequence of (4.21). Indeed, let us consider (4.8), with $\phi_{f}=e_{f, k+1}$. By the Poincaré inequality, we obtain

$$
\widehat{\alpha}_{f}\left\|e_{f, k+1}\right\|_{H^{1}\left(\Omega_{f}\right)}^{2} \leq\left\|\lambda_{k}\right\|_{\zeta}\left\|\gamma_{f} e_{f, k+1}\right\|_{\zeta}
$$

Exploiting inequality (4.16), we obtain that for all $k>0$ there exists a constant $K_{5}$ such that

$$
\left\|e_{f, k+1}\right\|_{H^{1}\left(\Omega_{f}\right)} \leq K_{5}\left\|\lambda_{k}\right\|_{\zeta}
$$

Proceeding in a similar way on (4.9) for $\phi_{w}=e_{w, k+1}$, it is possible to verify that for all $k>0$ there exists a constant $K_{6}$ such that

$$
\left\|e_{w, k+1}\right\|_{H^{1}\left(\Omega_{w}\right)} \leq K_{6}\left\|e_{f, k+1}\right\|_{H^{1}\left(\Omega_{f}\right)} .
$$

Now (4.22) follows from (4.21).

Remark 4.3. A different convergence proof could be carried out following the approach of [17] for proving the convergence of the so-called "Robin-Robin" scheme for the Poisson problem.

Indeed, let us consider (4.8) with $\phi_{f}=e_{f, k+1}$ :

$$
\widehat{a}_{f}\left(e_{f, k+1}, e_{f, k+1}\right)+\left(e_{f, k+1}, e_{f, k+1}\right)_{\zeta}=\left(\lambda_{k}, e_{f, k+1}\right)_{\zeta} .
$$

As already pointed out, if $\zeta>0$ a.e. on $\Gamma,\|\cdot\|_{\zeta}$ is a norm, thus by means of the Cauchy-Schwarz inequality, we obtain

$$
\widehat{a}_{f}\left(e_{f, k+1}, e_{f, k+1}\right)+\frac{1}{2}\left\|e_{f, k+1}\right\|_{\zeta}^{2} \leq \frac{1}{2}\left\|e_{w, k}\right\|_{\zeta}^{2} .
$$

Similarly, considering equation (4.9) with $\phi_{w}=e_{w, k+1}$ and applying the CauchySchwarz inequality, we obtain

$$
\widehat{a}_{w}\left(e_{w, k+1}, e_{w, k+1}\right)+\frac{1}{2}\left\|e_{w, k+1}\right\|_{\zeta}^{2} \leq \frac{1}{2}\left\|e_{f, k+1}\right\|_{\zeta}^{2} .
$$

Therefore,

$$
\widehat{a}_{f}\left(e_{f, k+1}, e_{f, k+1}\right)+\widehat{a}_{w}\left(e_{w, k+1}, e_{w, k+1}\right)+\frac{1}{2}\left\|e_{w, k+1}\right\|_{\zeta}^{2} \leq \frac{1}{2}\left\|e_{w, k}\right\|_{\zeta}^{2} .
$$

Summing up in the previous inequality from $k=0$ to $k=M$ we obtain

$$
\sum_{k=0}^{M}\left[\widehat{a}_{f}\left(e_{f, k+1}, e_{f, k+1}\right)+\widehat{a}_{w}\left(e_{w, k+1}, e_{w, k+1}\right)\right]+\frac{1}{2}\left\|e_{w, M}\right\|_{\zeta}^{2} \leq \frac{1}{2}\left\|e_{w, 0}\right\|_{\zeta}^{2},
$$

and owing to the coercivity of $\widehat{a}_{f}(\cdot, \cdot)$ and $\widehat{a}_{w}(\cdot, \cdot)$, we have

$$
\sum_{k=0}^{M}\left[\widehat{\alpha}_{f}\left\|e_{f, k+1}\right\|_{H_{\partial \Omega_{f} \backslash \Gamma}^{1}\left(\Omega_{f}\right)}^{2}+\widehat{\alpha}_{w}\left\|e_{w, k+1}\right\|_{H_{\partial \Omega_{w} \backslash \Gamma}^{1}\left(\Omega_{w}\right)}^{2}\right] \leq \frac{1}{2}\left\|e_{w, 0}\right\|_{\zeta}^{2} .
$$

Passing to the limit $M \rightarrow \infty$, we find that the series

$$
\sum_{k=0}^{\infty}\left[\widehat{\alpha}_{f}\left\|e_{f, k+1}\right\|_{H_{\partial \Omega_{f} \backslash \Gamma}^{1}\left(\Omega_{f}\right)}^{2}+\widehat{\alpha}_{w}\left\|e_{w, k+1}\right\|_{H_{\partial \Omega_{w} \backslash \Gamma}^{1}}^{2}\left(\Omega_{w}\right)\right]
$$

is convergent, and thus $\left\{e_{f, k+1}\right\} \rightarrow 0$ as $k \rightarrow \infty$ in the norm of $H_{\partial \Omega_{f} \backslash \Gamma}^{1}\left(\Omega_{f}\right)$ and $\left\{e_{w, k+1}\right\} \rightarrow 0$ as $k \rightarrow \infty$ in the norm of $H_{\partial \Omega_{w} \backslash \Gamma}^{1}\left(\Omega_{w}\right)$. However, no information on the error reduction rate at each step is deduced, as it was instead possible from Lemma 4.1. 


\subsection{Algorithmical issues.}

Convergence of the finite element scheme. In order to carry out a Galerkin finite element discretization of Problem 4.1, a triangulation $\mathcal{T}_{h}$ of $\Omega$ is introduced. It is supposed to be regular; i.e., the ratio between the external diameter of each element of the triangulation and the inner one is bounded - see [24]. Then we introduce a couple of finite dimensional spaces, $V_{h, f} \subset H_{\partial \Omega_{f} \backslash \Gamma}^{1}$ and $V_{h, w} \subset H_{\partial \Omega_{w} \backslash \Gamma}^{1}$, and denote by $N_{f}$ the dimension of $V_{h, f}$ and by $N_{w}$ the dimension of $V_{h, w}$. Let $\left\{\phi_{i, f}\right\}\left(i=1,2, \ldots, N_{f}\right)$ be a basis for $V_{h, f}$ and similarly $\left\{\phi_{i, w}\right\}\left(i=1,2, \ldots, N_{w}\right)$ be a basis for $V_{h, w}$. In what follows, the subscript $h$ will identify the space discrete solution (i.e., $C_{h f}$ stands for the discrete counterpart of $C_{f}$, and so on).

Then, the fully discrete form of the (4.5) and (4.6) reads (the dependence on the time index $n+1$ is still understood) as follows.

Problem 4.2. Given $C_{h w, 0}$, for $k=0,1, \ldots$, find $C_{h f, k+1} \in V_{h, f}$ and $C_{h w, k+1} \in$ $V_{h, w}$ such that for all $\phi_{i, f} \in V_{h, f}$ and $\phi_{i, w} \in V_{h, w}$

$$
\begin{aligned}
\widehat{a}_{f}\left(C_{h f, k+1}, \phi_{i, f}\right)+\left(C_{h f, k+1}, \phi_{i, f}\right)_{\zeta} & =\chi\left(C_{f}^{n}, \phi_{i, f}\right)+\left(C_{h w, k}, \phi_{i, f}\right)_{\zeta}+\left(f_{f}, \phi_{i, f}\right), \\
\widehat{a}_{w}\left(C_{h w, k+1}, \phi_{i, w}\right)+\left(C_{h w, k+1}, \phi_{i, w}\right)_{\zeta} & =\chi\left(C_{w}^{n}, \phi_{i, w}\right)+\left(C_{h f, k+1}, \phi_{i, w}\right)_{\zeta}+\left(f_{w}, \phi_{i, w}\right) .
\end{aligned}
$$

Owing to the inclusions $V_{h, f} \subset H_{\partial \Omega_{f} \backslash \Gamma}^{1}\left(\Omega_{f}\right)$ and $V_{h, w} \subset H_{\partial \Omega_{w} \backslash \Gamma}^{1}\left(\Omega_{w}\right)$, the convergence proof of the iterative method can be applied as well to the space discretized counterpart. Moreover, for any given solution $\mathbf{u}$ of the Navier-Stokes problem, such that $\zeta$ and $\boldsymbol{\mu}_{f}$ are specified, the constant $K$, introduced in Lemma 4.1 and representing the error reduction at each iteration, does not depend on $V_{h, f}$ and $V_{h, w}$; i.e., it does not depend on the mesh size $h$. Numerical results, indeed, confirm that the rate of convergence of the iterative scheme is essentially independent of $h$ (see Table 4.1).

TABLE 4.1

Comparison of the number of iterations to reach convergence, defined by test (4.4) with $\epsilon=10^{-8}$ for different grids. For these tests $\Omega_{f}=(0,4) \times(0,1), \Omega_{w}=(0,4) \times(-1,0), u_{x}=4 u_{0}(1-y) y, u_{y}=0$, $\mu_{f}=\mu_{w}=1.0 \mathrm{~cm}^{2} \mathrm{~s}^{-1}$ and $\zeta=10^{-1} \mathrm{~cm} \mathrm{~s}^{-1}$ (A) (values ${ }^{*}$ denotes the pure Galerkin method) $\mu_{f}=\mu_{w}=10^{-3} \mathrm{~cm}^{2} \mathrm{~s}^{-1}$ and $\zeta=10^{-1} \mathrm{~cm} \mathrm{~s}^{-1}$ (B) $\mu_{f}=\mu_{w}=10^{-3} \mathrm{~cm}^{2} \mathrm{~s}^{-1}$ and $\zeta=10^{-4} \mathrm{~cm} \mathrm{~s}^{-1}$ (C-physiologically relevant range). Finer grids are obtained by means of a uniform refinement and a regularization, thus we can say that $N=O\left(h^{-2}\right)$. For large values of $\zeta$ the coupled problem is severely ill-conditioned, yet the number of iterations is asymptotically independent of $h$.

\begin{tabular}{|c|c|c|c|c|}
\hline$h$ & $N$ & num.iter.(A) & num.iter.(B) & num.iter.(C) \\
\hline 0.1 & 4000 & $2^{*}$ & 3 & 1 \\
\hline 0.05 & 16000 & $2^{*}$ & 4 & 1 \\
\hline 0.025 & 60000 & $2^{*}$ & 5 & 2 \\
\hline 0.01875 & 106000 & $2^{*}$ & 5 & 2 \\
\hline 0.012 & 260000 & $2^{*}$ & $6-6^{*}$ & 2 \\
\hline
\end{tabular}

Introduction of a relaxation technique. In order to accelerate the convergence of the iterative method (4.5)-(4.6) it is possible to introduce an interface relaxation parameter $\theta \in \mathbb{R}^{+}$, leading to a relaxed Robin-Robin iterative method. Its convergence analysis is a straightforward generalization of the one that we have carried out in section 4.3. A suitable strategy to make an optimal choice of $\theta$ will be addressed in a forthcoming paper (see [23]).

We just point out that for the test cases considered here the number of iterations necessary for the convergence of the unrelaxed Robin-Robin scheme is already small even without relaxation (see Table 4.1). 
Convergence of the stabilized scheme. As previously pointed out, the adoption of stabilization techniques is often mandatory in the fluid domain. In a very general framework, this amounts to replace the bilinear form $\widehat{a}_{f}(\cdot, \cdot)$ introduced in (4.1) by (we omit the specification $f$ on the unknown $C$ for the sake of simplicity)

$$
\widehat{a}_{f, \text { stab }}(C, \phi)=\widehat{a}_{f}(C, \phi)+a_{f, h}(C, \phi),
$$

where $a_{f, h}(C, \phi)$ depends on the specific stabilization method at hand.

Let us assume for simplicity that $\boldsymbol{\mu}_{f}$ is symmetric and denote by

$$
L_{s} C=-\nabla \cdot \boldsymbol{\mu}_{f} \nabla C, \quad L_{s s} C=\frac{1}{2}(\nabla \cdot \mathbf{u}) C+\frac{1}{2} \mathbf{u} \cdot \nabla C
$$

the symmetric and the skew-symmetric parts of the fluid differential operator $L_{f}$ (note that $L_{f}=L_{s}+L_{s s}$ ). Then a common strongly consistent stabilization approach resorts to set

$$
a_{f, h}(C, \phi)=\sum_{K \in \mathcal{T}_{h}} \delta\left(L C, \frac{h_{K}}{|\mathbf{u}|}\left(L_{s s}+\kappa L_{s}\right) \phi\right)_{K},
$$

where $K$ is the current element of the triangulation $\mathcal{T}_{h}, h_{K}$ is its diameter, $(\cdot, \cdot)_{K}$ denotes the $L^{2}(K)$ scalar product, and $\delta$ is a positive parameter.

The SUPG method corresponds to set $\kappa=0$, while the Galerkin least squares (GaLS) method to $\kappa=1$. In both cases, if $\delta$ is suitably chosen, the stabilized bilinear form $\widehat{a}_{f, \text { stab }}(\cdot, \cdot)$ is coercive, the coercivity constant being independent of $h$ (see [24, Proposition 8.4.1]). Observe, however, that since we are using piecewise linear finite elements, the two methods actually coincide, since $L_{s}$ is null on linear functions.

On the ground of these properties we are going to prove that the rate of convergence of the iterative subdomain method remains independent of the mesh size $h$ also in the stabilized case.

First of all, let us reformulate the stabilized problem in the framework introduced in (4.10) and (4.11). Let $\mathcal{R}_{f \text {,stab }}: \Lambda \rightarrow \Lambda$ be the following operator: $\mathcal{R}_{f, \text { stab }} \psi=\gamma_{f} u_{f}$, where $u_{f} \in H_{\partial \Omega_{f} \backslash \Gamma}^{1}\left(\Omega_{f}\right)$ satisfies

$$
\widehat{a}_{f, \text { stab }}\left(u_{f}, \phi_{f}\right)+\left(u_{f}, \phi_{f}\right)_{\zeta}=\left(\psi, \phi_{f}\right)_{\zeta} \quad \forall \quad \phi_{f} \in H_{\partial \Omega_{f} \backslash \Gamma}^{1}\left(\Omega_{f}\right) .
$$

The operator $\mathcal{R}_{w}$ is unchanged.

We have the following result, which is the counterpart of Lemma 4.1.

LEMma 4.2. There exists a constant $K_{\text {stab }}<1$ depending on $\Omega_{f}, \Omega_{w}$, and $\zeta$, but independent of $h$, such that

$$
\|T \psi\|_{\zeta} \leq K_{\mathrm{stab}}\|\psi\|_{\zeta} \quad \forall \quad \psi \in \Lambda
$$

Proof. The proof can be carried along the same line of the proof of Lemma 4.1, provided we replace $\mathcal{R}_{f}$ by $\mathcal{R}_{f, \text { stab }}$. In particular, instead of (4.14) we find that

$$
\left\|\mathcal{R}_{f, \text { stab }} \psi\right\|_{\zeta} \leq K_{f, \text { stab }}\|\psi\|_{\zeta} \quad \forall \quad \psi \in \Lambda
$$

with

$$
K_{f, \mathrm{stab}}=\frac{K_{2}}{K_{2}+\widehat{\alpha}_{f, \mathrm{stab}}},
$$


and $\widehat{\alpha}_{f, \text { stab }}$ is the coercivity constant of the stabilized bilinear form $\widehat{a}_{f, \text { stab }}(\cdot, \cdot)$. The constant $K_{f \text {,stab }}$ is independent of $h$, as both $K_{2}$ and $\widehat{\alpha}_{f \text {,stab }}$ are independent of $h$.

The proof can now be completed by repeating exactly the subsequent steps of the proof of Lemma 4.1, obtaining eventually

$$
K_{\text {stab }}=\frac{K_{4}}{K_{4}+\widehat{\alpha}_{w}} K_{f, \text { stab }} .
$$

The limit (4.21) therefore holds also in the stabilized case; hence we can conclude with the following counterpart of Corollary 4.1.

COROLlary 4.2. The stabilized scheme is convergent, and its rate of convergence is independent of $h$.

As in the proof of Corollary 4.1, the constants $K_{5}$ and $K_{6}$ are independent of $h$, which ensures the independence of the rate of convergence of the mesh size.

A similar result can be also proved for the relaxed method (see [23]).

Stopping criterion. For Problem 4.1, a suitable test to verify whether the solution has converged is based on the (normalized) difference between the last two iterations in the fluid and the wall domain $\Omega_{f}$ and $\Omega_{w}$. Precisely, the stopping criterion reads

$$
\frac{\left\|C_{f, k+1}^{n+1}-C_{f, k}^{n+1}\right\|_{L^{2}\left(\Omega_{f}\right)}}{\left\|C_{f, k+1}^{n+1}\right\|_{L^{2}\left(\Omega_{f}\right)}}+\frac{\left\|C_{w, k+1}^{n+1}-C_{w, k}^{n+1}\right\|_{L^{2}\left(\Omega_{w}\right)}}{\left\|C_{w, k+1}^{n+1}\right\|_{L^{2}\left(\Omega_{w}\right)}} \leq \epsilon,
$$

where $\epsilon$ is the desired tolerance.

5. Test cases of physiological interest. In this section we apply the described models to a physiologically relevant test case. For this reason we consider a stenosed part of a large artery, that is, a vessel whose section has a narrowing restriction due to a disease of the arterial wall, such as atherosclerosis. The solute that we consider in this case is oxygen $\left(\mathrm{O}_{2}\right)$. A crucial issue is how to choose the functional dependence of $\zeta$ from $\boldsymbol{\sigma}(\mathbf{u})$ in Problems 2.2 and 2.3, in order to catch the mean oxygen flow through $\Gamma$ under a mean value of the shear stress. Following the suggestion of [27], we propose a linear relation of the form $\zeta=K_{1}+K_{2}|\boldsymbol{\sigma}(\mathbf{u})|$. At this stage, the issue is how to fix the constants $K_{1}$ and $K_{2}$. The mean oxygen flow through $\Gamma$ is defined by

$$
\phi_{\text {mean }}=\frac{1}{\operatorname{meas}(\Gamma)} \int_{\Gamma} \zeta(\boldsymbol{\sigma}(\mathbf{u}))\left(C_{f}-q_{w}\right) d \gamma,
$$

where $q_{w}=k_{w}$ in the wall-free model and $q_{w}=C_{w}$ in the free-wall model. A plausible value for $\phi_{\text {mean }}$, proposed in [27], is $4.82 \cdot 10^{-6} \mathrm{ml} \mathrm{cm}^{-2} \mathrm{~s}^{-1}$. On the other hand, again in [27], the mean oxygen concentration in the lumen can be set $C_{0}=$ $2.58 \cdot 10^{-3} \mathrm{ml} \mathrm{cm}^{-3}$. Next we approximate, under integration in (5.1), $\boldsymbol{\sigma}(\mathbf{u})$ by $\boldsymbol{\sigma}_{0}=$ $1.98 \mathrm{dyn}_{\mathrm{cm}}^{-2}$ (calculated from the Poiseuille flow in a channel with the same diameter and the same inflow conditions as the ones considered in $\left.\Omega_{f}\right)$ and obtain $\phi_{\text {mean }} \simeq$ $\left(K_{1}+K_{2} \sigma_{0}\right) \cdot 0.3 C_{0}$, where we have made the approximation

$$
\frac{1}{\operatorname{meas}(\Gamma)} \int_{\Gamma}\left(C_{f}-q_{w}\right) d \gamma \simeq 0.3 C_{0}
$$

(which is plausible at equilibrium). Finally it is assumed that $K_{1}=K_{2} \boldsymbol{\sigma}_{0} / 2$, and henceforth we obtain $\phi_{\text {mean }}=\frac{3}{2} K_{2} \sigma_{0} \cdot 0.3 C_{0}$; thus $K_{2}=1.57 \cdot 10^{-4} \mathrm{~cm}^{3} \mathrm{dyn}^{-1} \mathrm{~s}^{-1}$ and $K_{1}=3.11 \cdot 10^{-3} \mathrm{~cm} \mathrm{~s}^{-1}$. For other kind of solutes, such as macromolecules, e.g., lipoproteins, in vivo measurements suggest that a nonlinear dependence of $\zeta$ from $\boldsymbol{\sigma}(\mathbf{u})$ should be introduced (see [26]). 
5.1. Description of the domain and of the data. Figure 5.1 shows a sketch of the domain $\Omega_{f}$ and $\Omega_{w}$. Domain $\Omega_{f}$ is symmetric with respect to its longitudinal axis. The interface $\Gamma$ and the lower side $\Gamma_{5}$ are defined by the following functions:

$$
\begin{gathered}
\Gamma:\left\{\begin{array}{l}
0 \leq x<l_{1}, y=0, \\
l_{1} \leq x<l_{2}, y=(T / 2)\left(1+\cos 2 \pi\left(x-\left(l_{1}+l / 2\right) / l\right)\right), \\
l_{2} \leq x<L, y=0,
\end{array}\right. \\
\Gamma_{5}:\left\{\begin{array}{l}
0 \leq x<l_{1}, y=y_{\Gamma}-w_{1}, \\
l_{1} \leq x<l_{1}+l / 2, y=y_{\Gamma}-\left[w_{1}+2\left(w_{2}-w_{1}\right)\left(x-l_{1}\right) / l\right], \\
l_{1}+l / 2 \leq x<l_{2}, y=y_{\Gamma}-\left[w_{1}+2\left(w_{2}-w_{1}\right)\left(1-\left(x-l_{1}\right) / l\right)\right], \\
l_{2} \leq x<L, y=y_{\Gamma}-w_{1},
\end{array}\right.
\end{gathered}
$$

where $L=10 \mathrm{~cm}$ is the total length of the domain; $R=0.5 \mathrm{~cm}$ is the radius of the lumen; $l_{1}=4 \mathrm{~cm}$ and $l_{2}=5 \mathrm{~cm}$ are the abscissa defining the stenosed region, $l=l_{2}-l_{1}$; $T=R / 2$ defines the narrowing rate; and $y_{\Gamma}$ stands for the corresponding value on $\Gamma$. Finally the minimal and maximal wall thicknesses are equal to $8 \%$ and $12 \%$ of the radius of the lumen, respectively. The domain $\Omega_{f}$ is discretized by 18728 nodes and $\Omega_{w}$ by 6100 nodes. For the time discretization we consider $\Delta t=0.01 \mathrm{~s}$.

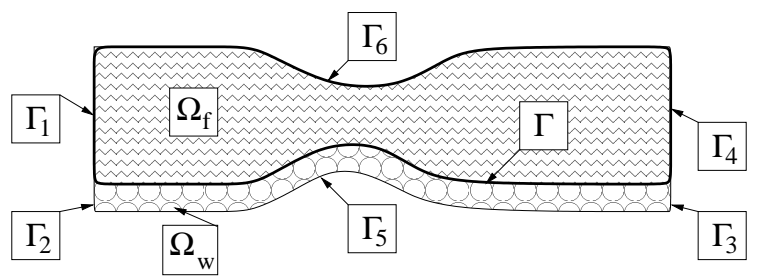

FIG. 5.1. Simplified scheme of the domains $\Omega_{f}$ and $\Omega_{w}$.

We choose the following data for the Navier-Stokes equations (see Figure 5.1 for the boundary definition):

$$
\begin{aligned}
u_{x} & =u_{0}\left(1-((y-R) / R)^{2}\right) & & \text { on } \Gamma_{1}, \\
u_{y} & =0 & & \text { on } \Gamma_{1}, \\
(-\nu \nabla \mathbf{u}+P \mathbf{I}) \cdot \mathbf{n} & =0 & & \text { on } \Gamma_{4}, \\
\mathbf{u} & =0 & & \text { on } \Gamma \text { and on } \Gamma_{6},
\end{aligned}
$$

where $u_{0}=15 \mathrm{~cm} \mathrm{~s}^{-1}$ is the maximal inlet velocity and the kinematic viscosity is $\nu=0.033 \mathrm{~cm}^{2} \mathrm{~s}^{-1}$. Moreover, we consider a null velocity field on $\overline{\Omega_{f}} \backslash \Gamma_{1}$ at time $t=0$. For the wall-free model we define a normalized concentration $C_{f}=C_{f}^{\prime} / C_{0}$, where $C_{f}^{\prime}$ is the concentration of oxygen in the vessel and $C_{0}=2.58 \cdot 10^{-3} \mathrm{ml} \mathrm{cm}{ }^{-3}$ is a reference concentration. For the concentration $C_{f}$ we consider the following data: $C_{f}=1.0$ on $\Gamma_{1},\left(\boldsymbol{\mu}_{f} \nabla C_{f}\right) \cdot \mathbf{n}_{f}=0$ on $\Gamma_{4}$, condition (2.2a) on $\Gamma$ with $\kappa_{w}=0.5$. Moreover we consider a diffusivity $\boldsymbol{\mu}_{f}$ independent of $\mathbf{d}$. Indeed, for the sake of simplicity we choose $\mathcal{D}=0$ in $(2.3)$, and thus $\boldsymbol{\mu}_{f}=\mu_{f, 0} \mathbf{I}$ with $\mu_{f, 0}=5 \cdot 10^{-5} \mathrm{~cm}^{2} \mathrm{~s}^{-1}$. Nevertheless, some tests made in order to evaluate the dependence of $C_{f}$ on the shear rate through the diffusivity $\boldsymbol{\mu}_{f}$ show that nonnull values of $\mathcal{D}$, taken, for instance, from [12], may induce changes on $C_{f}$ up to $7 \%$ with respect to the case $\mathcal{D}=0$, where the shear rate and the concentration gradients are high. Finally we set initial condition $C_{f}=1.0$ in $\Omega_{f}$. For the fluid-wall model in $\Omega_{f}$ we consider the same data except from the 
condition on $\Gamma$ that is represented by (2.7). For the diffusion problem in $\Omega_{w}$ we consider a normalized concentration $C_{w}=C_{w}^{\prime} / C_{0}$ and the following boundary and initial data: $C_{w}=0.5$ on $\Gamma_{2}$ and $\mu_{w} \nabla C_{w} \cdot \mathbf{n}_{w}=0$ on $\Gamma_{3}$, and $\mu_{w} \nabla C_{w} \cdot \mathbf{n}_{w}=0$ or $C_{w}=0.5$ on $\Gamma_{5}$, interface condition (2.6) on $\Gamma$, initial condition $C_{w}=0.5$ in $\Omega_{w}$. Finally, because oxygen is a very little molecule, it is reasonable to choose $\mu_{w}=\mu_{f, 0}$.

5.2. Numerical results. The aim of this numerical investigation is to compare the predictions given by the wall-free and the fluid-wall models on the oxygenation of the wall. For this reason we compare the two models under very simple flow conditions, such as stationary blood flow. Consequently, we compute at first the stationary flow in the stenosed domain. This calculation includes the computation at each time step $t^{n}$ of the rate of deformation tensor $\mathbf{d}=1 / 2\left(\nabla \mathbf{u}+\nabla \mathbf{u}^{T}\right)$ as well as the local stress tensor $\mathbf{T}=2 \nu \mathbf{d}$ and the shear stress $\boldsymbol{\sigma}(\mathbf{u})=\boldsymbol{\tau} \cdot \mathbf{T} \cdot \mathbf{n}$ on $\Gamma$. These quantities are computed by means of a variational recovering technique, through an $H^{1}$-projection of $\mathbf{d}$ (and consequently $\mathbf{T}$ ) on the subspace of piecewise linear finite elements in $\Omega_{f}$. The shear stress $\boldsymbol{\sigma}(\mathbf{u})$ is then computed on the nodes lying on $\Gamma$. Then, given $\mathbf{u}, P$ on $\Omega_{f}$, and $\boldsymbol{\sigma}(\mathbf{u})$ on $\Gamma$, we can solve the advection-diffusion problem.

5.2.1. Solution of the Navier-Stokes equations. The blood flow in this test case features a Reynolds number $R e=2 R|\overline{\mathbf{u} \mid}|_{\Gamma_{1}} / \nu$ small enough $(=300)$ to allow a stationary solution of the Navier-Stokes equations. The stationary state (defined by the test $\left\|\mathbf{u}^{n}-\mathbf{u}^{n-1}\right\|_{L^{2}} /\left\|\mathbf{u}^{n}\right\|_{L^{2}}<10^{-4}$ ) is satisfied, starting from null initial velocity, after $9 s$. The steady flow field shows thin boundary layers upstream the minimal section point and a large recirculation zone downstream it. As it will be clear later, these phenomena have a direct effect on the concentration of solute in the lumen and in the arterial wall.

5.2.2. Solution of the advection-diffusion equations. Figures $5.2,5.3$, and 5.4 show the results of some simulations concerning the wall-free and the fluid-wall models. First of all, we observe that these models give similar results about the solute dynamics in the blood. In particular we notice that, according to (2.7) the difference in the initial concentrations $\left(\left(C_{0, f}-k_{w}\right)>0\right.$ or $\left.\left(C_{0, f}-C_{0, w}\right)>0\right)$ causes a flux of solute leaving $\Omega_{f}$, consequently the concentration near the arterial wall is slightly lower than the mean value $C_{f}=1$. Moreover, the solute flux across the arterial wall is higher in the upstream side of the stenosis because of two phenomena: in the upstream part of the stenosis the blood pushes the solute towards the wall; consequently a greater amount of solute passes from the blood to the arterial wall. These phenomena are magnified by the dependence of the wall permeability $\zeta$ on the shear stress induced in the arterial wall. In fact in the upstream side of the stenosis the shear stress, and consequently the wall permeability, is high; thus the solute flux towards the arterial wall is increased. On the other hand, downstream the minimal span of the vessel the blood flow detaches from the wall and a wide recirculation zone is generated (see Figures 5.5 and 5.2). In the recirculation the blood velocity is highly reduced; consequently the fluid layers with low solute concentration next to the arterial wall are hardly removed. Moreover, the shear stress induced on the wall is low and so is the wall permeability. For these reasons the solute flux leaving the vessel in the downstream side of the stenosis is sensibly lower than the one in the upstream side.

5.2.3. Comparison of the wall-free and fluid-wall model. Although giving the same qualitative results on the solute dynamics in blood, the wall-free and the 


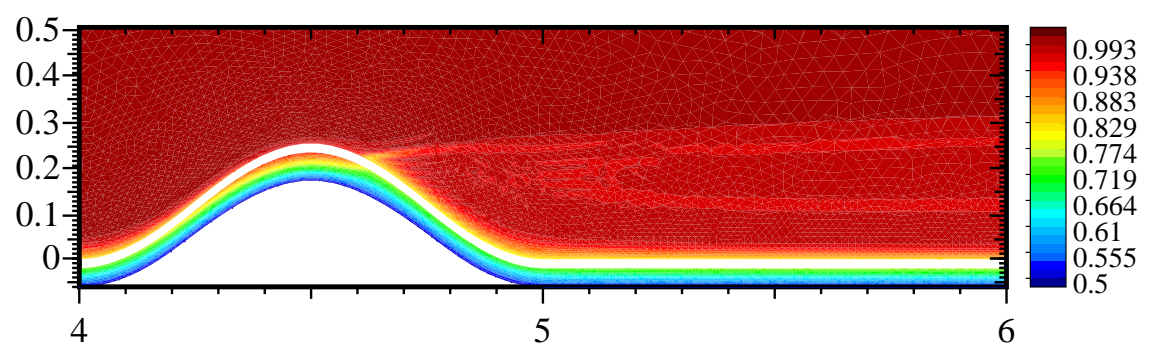

FIG. 5.2. Concentration field in the lumen and in the arterial wall at the stationary state (after $54.21 s)$, computed with the fluid-wall model with Dirichlet boundary conditions on $\Gamma_{5}$. Domains $\Omega_{f}$ and $\Omega_{w}$ are not represented as contiguous, in order to put the interface $\Gamma$ into evidence.
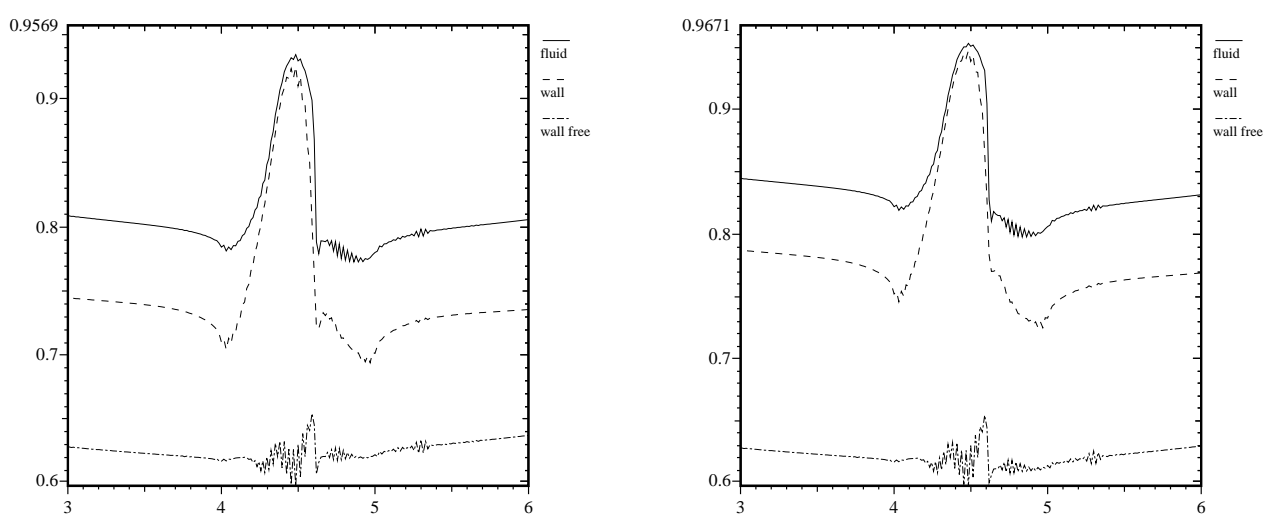

FIG. 5.3. Comparison of solute concentration on the wall boundary for wall-free model (dasheddotted line) and fluid-wall model (solid and dashed lines) at time $t=10 \mathrm{~s}$ (left) and $t=100 \mathrm{~s}$ (right).

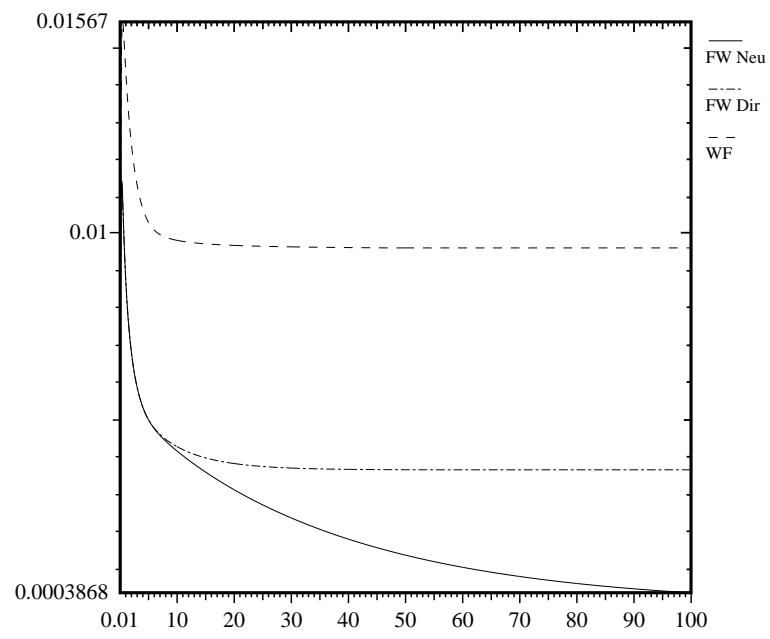

FIG. 5.4. Total flux of solute across $\Gamma\left(\mathrm{cm}^{2} \mathrm{~s}^{-1}\right)$ for the wall-free (WF) and the fluid-wall model, with Dirichlet boundary conditions (FW Dir) and homogeneous Neumann boundary conditions (WF $\mathrm{Neu}$ ) on $\Gamma_{5}$. The wall-free model reaches stationary state after $47.65 \mathrm{~s}$ while the fluid-wall model with Dirichlet boundary conditions reaches stationary state after $54.21 \mathrm{~s}$. The fluid wall model with Neumann boundary conditions doesn't reach stationary state after 100 s. 

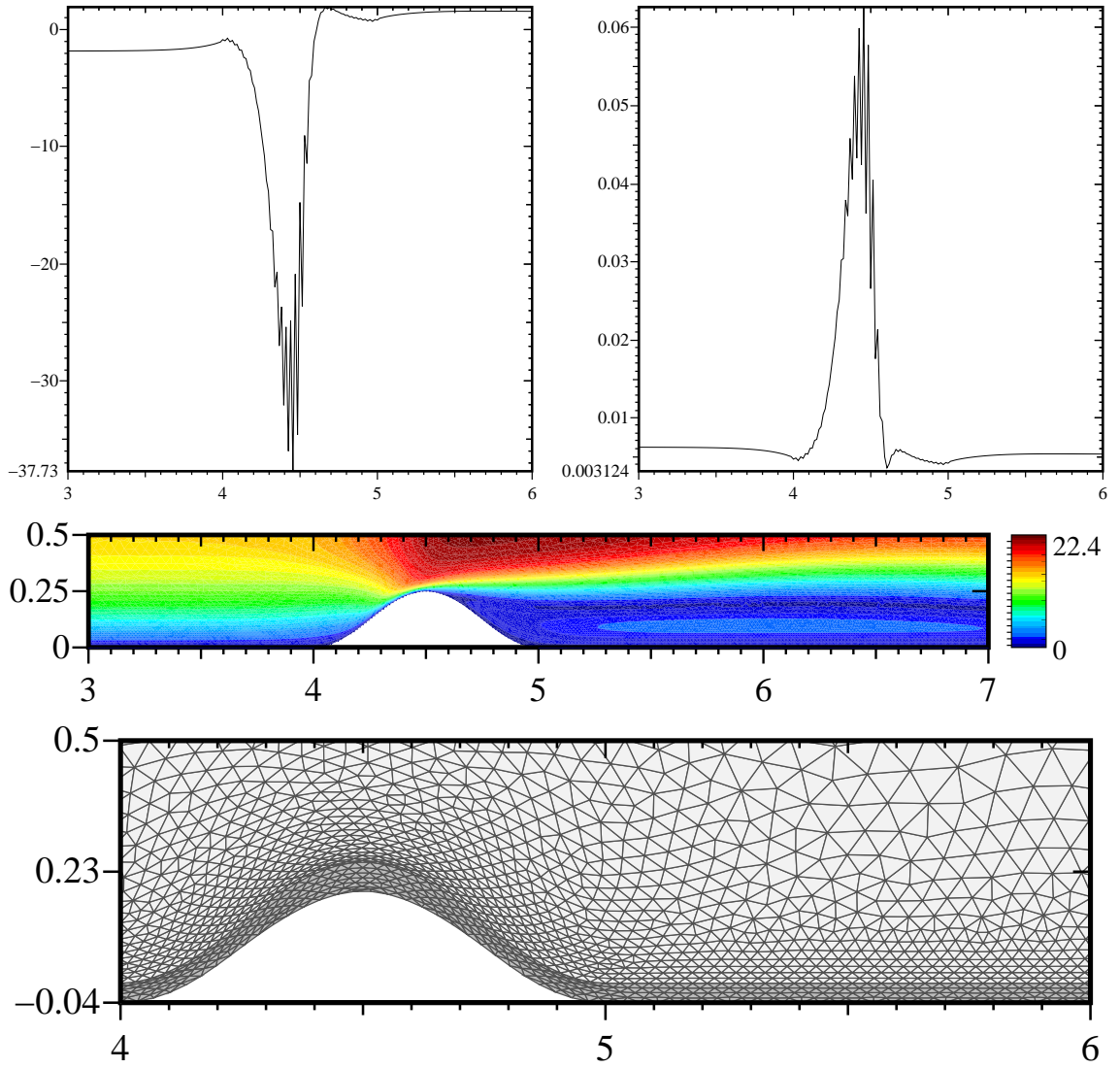

FIG. 5.5. Shear stress on $\Gamma\left(\right.$ dyn $\left.\mathrm{cm}^{-2}\right)$ (top-left); arterial wall permeability $\left(\mathrm{cm} \mathrm{s}^{-1}\right)$ (topright); a detail of $|\mathbf{u}|$ at the stationary state (middle); a detail of the discretization of $\Omega_{f}$ and $\Omega_{w}$ into triangles (bottom).

fluid-wall models show some relevant differences, in particular concerning the dynamics of the wall absorption. This is mainly due to the fact that the fluid-wall model takes into account the accumulation of solute in the arterial wall. For example, according to (2.7), the solute flux towards the arterial wall decreases as far as the wall concentration increases. For this reason, the profile of the concentration $C_{f}$ along the arterial wall for the fluid-wall case is slightly higher than the wall-free one. Consequently, we point out that the difference between the blood concentration profiles for these two models becomes larger in the later time steps because the wall concentration is gradually increasing. Finally, let us compare the predictions of the two models on the total solute flux leaving $\Omega_{f}$ across $\Gamma$, that corresponds, when the considered solute is oxygen, to the oxygenation of the wall. To this aim, we refer to Figure 5.4, where we plot the function $\phi(t)=\int_{0}^{\Gamma} \zeta\left(C_{f}-q_{w}\right)$ (where $q_{w}=k_{w}$ in the wall-free model and $q_{w}=C_{w}$ in the free-wall model) for different boundary conditions. We notice that the two models yield different asymptotic value of the flux. More precisely, the wall oxygenation is higher for the wall-free model, and it quickly reaches a steady-state constant value. On the other hand, taking into account the saturation of the concentration in the wall, the fluid-wall model predicts a lower flux, and a much longer time is necessary to reach the steady state for the solute exchanges between lumen and 
wall. We also point out that the results of the fluid-wall model are influenced by the boundary conditions chosen on $\Gamma_{5}$. Homogeneous Neumann conditions will induce a relevant accumulation of solute in $\Omega_{w}$; on the other hand, Dirichlet conditions will reduce this phenomenon, providing a solute flux outgoing $\Gamma_{5}$. Consequently, the dynamics of solute exchanges between $\Omega_{f}$ and $\Omega_{w}$ are directly influenced by boundary conditions on $\Gamma_{5}$. A detailed discussion of the choice of these conditions, based on an experimental validation would be worthwhile to justify the physiological relevance of the results given by the fluid-wall model. In any case, we can assert that no matter which coefficients $\left(\boldsymbol{\mu}_{f}, \mu_{w}, \zeta\right)$ are used, the wall-free and the fluid-wall models show a different dynamical behavior with respect to time. Actually, the interpretation of Figure 5.4 in a quantitative sense is not particularly significant, since it is influenced by the specific choice of the dependence of $\zeta$ on the shear rate. In this respect, more refined experimental estimates of $K_{1}$ and $K_{2}$ (and, in general, of $\zeta$ ) are required to allow precise physiological evaluations. However, even from their merely qualitative interpretation, these results show that a more sophisticated model for the arterial wall may substantially affect the analysis of absorption dynamics of solutes. In the case of oxygen, this may be quite relevant in the investigation of pathologies of the arterial tree, such as hypoxia.

\section{REFERENCES}

[1] D.A. Baker, J.E. Holte, And S.V. Patankar, Computationally two-dimensional finitedifference model for hollow-fibre blood-gas exchange devices, Med. Biol. Engrg. Comput., 29 (1991), pp. 482-488.

[2] C.G. Caro, J.M. Fitzgerald, and R.C. Schroter, Atheroma and wall shear: Observation, correlation and proposal of a shear dependent mass transfer mechanism of atherogenesis, Proc. Roy. Soc. London Ser. A, 177 (1971), pp. 109-159.

[3] T.E. Diller, B.B. Mikic, ANd P.A. Drinker, Shear-induced augmentation of oxygen transfer in blood, J. Biomech. Engrg., 102 (1980), pp. 67-72.

[4] T.K. Goldstick And P.B. Dobrin, Arterial wall oxygen transport and its relationship to atherogenesis, in Handbook of Bioengineering, R. Skalak and S. Chen, eds., McGraw-Hill, New York, 1987, Chap. 22.

[5] J. HeYwood, The Navier-Stokes equations: On the existence, regularity and decay of solutions, Indiana Univ. Math. J., 29 (1980), pp. 639-681.

[6] J. Heywood And R. RANNACHER, Finite element approximation of the nonstationary NavierStokes problem. I. Regularity of solutions and second-order error estimates for spatial discretization, SIAM J. Numer. Anal., 19 (1982), pp. 275-311.

[7] E. Hopf, Über die Aufgangswertaufgabe fur die hydrodynamischen Grundliechungen, Math. Nachr., 4 (1951), pp. 213-231.

[8] J.R. Hughes and M. Mallet, A new finite element formulation for computational fluid dynamics. III. The generalized streamline operator for multidimensional advective-diffusive systems, Comput. Methods Appl. Mech. Engrg., 58 (1986), pp. 305-328.

[9] H. Jo, R.O. Dull, T.M. Hollis, and J.M. Tarbell, Endothelial albumin permeability is shear dependent, time dependent and reversible, Amer. J. Physiol., 260 (1991), pp. H1992-H1996.

[10] G. Karner and K. Perktold, Numerical modeling of mass transport in the arterial wall, in Proceedings of the 1999 Bioengineering Conference, BED, Vol. 42, V.K. Goal, R.L. Spilker, G.A. Ateshian, and L.J. Solawsky, eds., ASME, New York, 1999, pp. 739-740

[11] G. Karner, K. Perktold, H.-P. Zehentner, and M. Prosi, Mass transport in large arteries and through the arterial wall, in Intra and Extracorporeal Cardiovascular Fluid Dynamics, Vol. 2, Fluid-Structure Interaction, Adv. Fluid Mech. 23, P. Verdonck and K. Perktold, eds., WIT Press-Computational Mechanics, Southampton, Bellerica, MA, 2000, pp. 209-247.

[12] K.H. Keller, Effect of fluid shear on mass transport in flowing blood, Fed. Proc., 30 (1971), pp. 1591-1599.

[13] J. LeRAY, Etude de diverses équations intégrales et de quelques problèmes que pose l'hydrodynamique, J. Math. Pures Appl., 12 (1933), pp. 1-82.

[14] J. LeRAY, Essai sur les mouvement d'un liquide visqueux emplissant l'espace, Acta. Math., 63 (1934), pp. 193-248. 
[15] J. Leray, Essai sur les mouvements plans d'un liquide visqueux que limitent des parois, J. Math. Pures Appl., 13 (1934), pp. 331-418.

[16] J.L. Lions and E. Magenes, Problèmes aux Limites non Homogènes et Applications 1, Dunod, Paris, 1968.

[17] P.-L. Lions, On the Schwarz alternating method. III. A variant for nonoverlapping subdomains, in Third International Symposium on Domain Decomposition Methods for Partial Differential Equations, Houston, 1989, SIAM, Philadelphia, 1990, pp. 202-223.

[18] J.A. Moore And C.R. Ethier, Oxygen mass transfer calculations in large arteries, J. Biomech. Engrg., 119 (1997), pp. 469-475.

[19] W. Nichols and M. O'Rourke, eds., McDonald's Blood Flow in Arteries, 3rd ed., Edward Arnold, London, 1990.

[20] A. Quarteroni, F. Saleri, and A. Veneziani, Analysis of the Yosida method for the incompressible Navier-Stokes equations, J. Math. Pures Appl. (9), 78 (1999), pp. 473-503.

[21] A. Quarteroni, F. Saleri, and A. Veneziani, Factorization methods for the numerical approximation of the incompressible Navier-Stokes equations, Comput. Meth. Appl. Mech. Engrg., 188 (2000), pp. 505-526.

[22] A. Quarteroni, M. Tuveri, and A. Veneziani, Computational vascular fluid dynamics: Problems, models and methods, Comput. Visualisation Sci., 2 (2000), pp. 163-197.

[23] A. Quarteroni, A. Veneziani, And P. Zunino, A domain decomposition method for advectiondiffusion processes with application to blood solutes, SIAM J. Sci. Comput., to appear.

[24] A. Quarteroni and A. Valli, Numerical Approximation of Partial Differential Equations, Springer-Verlag, Berlin, 1994.

[25] A. Quarteroni and A. Valli, Domain Decomposition Methods for Partial Differential Equations, Oxford University Press, Oxford, 1999.

[26] G. Rappitsch and K. Perktold, Pulsatile albumin transport in large arteries: A numerical simulation study, J. Biomech. Engrg., 118 (1996), pp. 511-519.

[27] G. Rappitsch, K. Perktold, and E. Pernkopf, Numerical modelling of shear-dependent mass transfer in large arteries, Internat. J. Numer. Methods Fluids, 25 (1997), pp. 847857.

[28] R. Temam, Navier-Stokes Equations: Theory and Numerical Analysis, 3rd ed., North-Holland, Amsterdam, 1984

[29] R. Temam, Navier-Stokes Equations and Nonlinear Functional Analysis, SIAM, Philadelphia, 1983.

[30] N.L. WAng AND K.H. Keller, Solute transport induced by erythrocyte motions in shear flow, Trans. Amer. Soc. Artif. Intern. Organs, 25 (1979), pp. 14-17.

[31] N.L. Wang And K.H. Keller, Augmented transport of extracellular solutes in concentrated erythrocyte suspensions in Couette flow, J. Colloid Inter. Sci., 103 (1985), pp. 210-225. 